\title{
IL-13-induced airway mucus production is attenuated by MAPK13 inhibition
}

\author{
Yael G. Alevy, ${ }^{1}$ Anand C. Patel,1,2 Arthur G. Romero, ${ }^{1}$ Dhara A. Patel,, Jennifer Tucker, ${ }^{1}$ \\ William T. Roswit, ${ }^{1}$ Chantel A. Miller, ${ }^{1}$ Richard F. Heier, ${ }^{1}$ Derek E. Byers, ${ }^{1}$ \\ Tom J. Brett, ${ }^{1,3,4}$ and Michael J. Holtzman ${ }^{1,3}$
}

\author{
${ }^{1}$ Drug Discovery Program, Pulmonary and Critical Care Medicine, Department of Medicine, ${ }^{2}$ Department of Pediatrics, \\ ${ }^{3}$ Department of Cell Biology, and ${ }^{2}$ Department of Biochemistry, Washington University School of Medicine, St. Louis, Missouri, USA.
}

\begin{abstract}
Increased mucus production is a common cause of morbidity and mortality in inflammatory airway diseases, including asthma, chronic obstructive pulmonary disease (COPD), and cystic fibrosis. However, the precise molecular mechanisms for pathogenic mucus production are largely undetermined. Accordingly, there are no specific and effective anti-mucus therapeutics. Here, we define a signaling pathway from chloride channel calcium-activated 1 (CLCA1) to MAPK13 that is responsible for IL-13-driven mucus production in human airway epithelial cells. The same pathway was also highly activated in the lungs of humans with excess mucus production due to COPD. We further validated the pathway by using structure-based drug design to develop a series of novel MAPK13 inhibitors with nanomolar potency that effectively reduced mucus production in human airway epithelial cells. These results uncover and validate a new pathway for regulating mucus production as well as a corresponding therapeutic approach to mucus overproduction in inflammatory airway diseases.
\end{abstract}

\section{Introduction}

An excess of airway mucous secretions is likely one of the most common maladies of humankind. The condition is an invariable feature of acute respiratory illnesses and a characteristic feature of chronic lung diseases, such as asthma and chronic obstructive pulmonary disease (COPD). Indeed, mucus overproduction is likely responsible for much of the morbidity and mortality associated with all of these conditions. In the case of asthma, reports of mucus plugging and inspissation are typical of autopsies of patients with asthma (1). Similarly, much of the distress of patients with COPD may depend on disease of small airways that are overpopulated with mucous cells (2). Moreover, mucus production may be an early sign of a progressive decline in lung function in COPD (3). Excess mucus is likely due to increased biosynthesis and secretion of the secretory mucins (particularly MUC5AC and MUC5B) that are the major macromolecular constituents of airway mucus (4). At present, however, there is no specific and effective treatment for controlling overproduction of respiratory mucin or consequent airway mucus levels.

One of the chief reasons for the lack of effective therapeutics for excess mucus production is that the underlying cellular and molecular mechanism for this process is poorly understood. We reasoned that two basic questions must be resolved: first, what are the upstream extracellular events that drive a precursor epithelial cell to become a mucous cell, and second, what are the subsequent downstream signaling events that occur within the airway epithelial cell to drive mucin gene expression? For upstream events, other groups and ours have provided evidence that initial stimuli, such as allergens, viruses, and cigarette smoking, will lead to immune cell production of IL-13 as the critical driver for mucus production (5-8). Other laboratories and ours also have shown

Authorship note: Yael G. Alevy and Anand C. Patel contributed equally to this work. Conflict of interest: Michael J. Holtzman is the principal investigator for grants to Washington University from Hoffman-La Roche and Forest Laboratories Inc. Citation for this article: J Clin Invest. 2012;122(12):4555-4568. doi:10.1172/JCI64896. that the subsequent downstream events for IL-13 signaling in mucous precursor cells likely involve upregulation and activation of the IL-13 receptor and associated STAT6 transcription factor $(8,9)$. However, the next step between these events and downstream mucin gene expression still needed to be defined. The lack of identifiable STAT6-binding sites in the MUC5AC mucin gene promoter indicates that intermediate steps are required to convert the IL-13 signal to mucin gene expression $(10,11)$. In that regard, other studies of cultured human airway epithelial cells have suggested that activation of MEK1/2, PI3K, SPhk1, and MAPK14 (p38 $\alpha$-MAPK) are necessary for IL-13-induced mucus production $(12,13)$. However, these conclusions were typically based on the effects of chemical inhibitors at relatively high concentrations without target validation using genetic tools. Moreover, it remained uncertain whether these signaling events were associated with mucous cell metaplasia/hyperplasia and mucus overproduction in humans with lung disease.

In this context, we previously provided evidence that calcium-activated chloride channel (CLCA) genes may fulfill a critical role for the development of mucous cell metaplasia. For example, gene transfer with vectors encoding mouse Clca3, Clca5, or Clca6 is sufficient for airway mucus production in mice $(14,15)$. Furthermore, both the mouse Clca and human CLCA gene promoter regions contain consensus STAT6-binding sites that could mediate direct responsiveness to IL-13 stimulation (16). In addition, CLCA proteins undergo extracellular secretion and cleavage, suggesting that they might function as signaling molecules rather than ion channels $(17,18)$. In this work, we better defined the signal transduction basis for mucus production through the unexpected finding that human CLCA1 activates MAPK13 (also known as $\mathrm{p} 38 \mathrm{\delta}-\mathrm{MAPK}$ ), which in turn conveys a signal to stimulate MUC5AC mucin gene expression. We detected the same signaling pathway to be active in humans with COPD, providing a rationale for further therapeutic development. We used a drug design strategy that takes advantage of target homology to shift the activity profile of inhibitors from MAPK14 (19) toward 

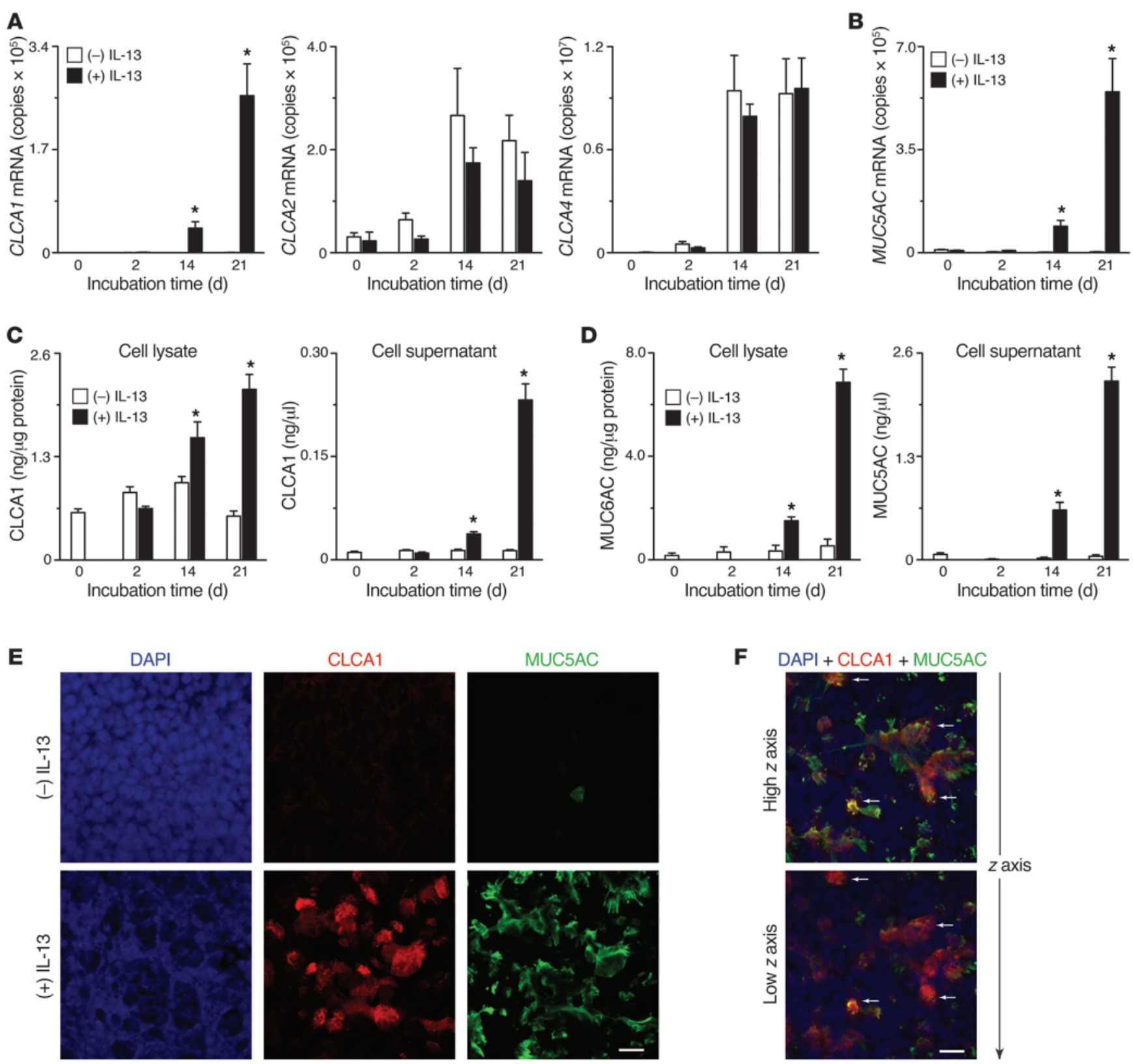

G

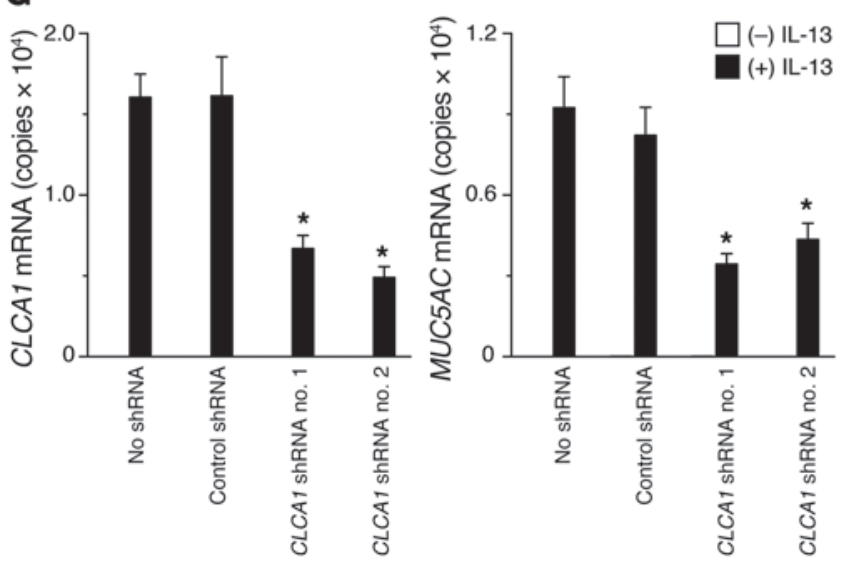

H

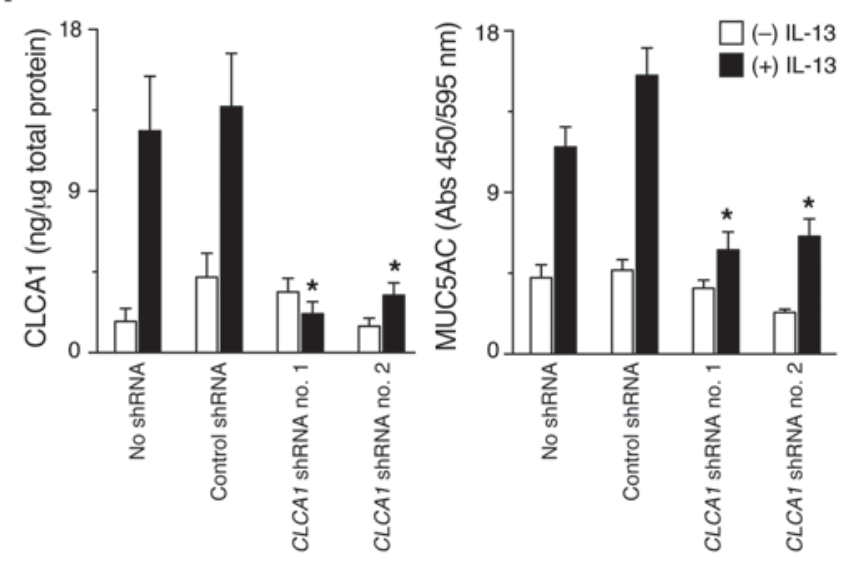




\section{Figure 1}

CLCA1 is necessary for IL-13-stimulated mucus production in human airway epithelial cells. (A) hTECs were incubated with IL-13 $(50 \mathrm{ng} / \mathrm{ml})$ under submerged conditions for 2 days and then air-liquid interface conditions for up to 21 days, and cell lysates were analyzed for CLCA1, CLCA2, and CLCA4 mRNA levels by real-time qPCR assay. CLCA3 mRNA was undetectable under these conditions (data not shown). (B) Corresponding levels of MUC5AC mRNA. (C) Corresponding levels of CLCA1 in cell lysate and apical cell supernatant determined by ELISA. (D) Corresponding levels of MUC5AC determined by ELISA. (E) Corresponding immunocytochemistry for DAPI, CLCA1, and MUC5AC using confocal microscopy. Scale bar: $50 \mu \mathrm{m}$. (F) Corresponding immunocytochemistry at a more apical (high $z$ axis) and subjacent (low $z$ axis) cellular location. Arrows indicate the same reference cells for high and low $z$ axis. Scale bar: $50 \mu \mathrm{m}$. (G) Levels of CLCA1 and MUC5AC mRNA in hTECs that were transduced with lentivirus encoding CLCA1 or control shRNA. (H) For conditions in E, corresponding cellular levels of CLCA1 and MUC5AC by ELISA. All values represent mean \pm SEM. ${ }^{\star} P<0.05$, increase from corresponding no IL-13 treatment control or decrease from no shRNA treatment.

increased activity against MAPK13 and found that these novel compounds effectively block IL-13-stimulated mucus production in human airway epithelial cells. The results thereby validate a novel therapeutic approach to hypersecretory diseases of the pulmonary airways and perhaps other sites as well.

\section{Results}

CLCA1 controls mucin gene expression. The mouse Clca gene family contains at least 7 members, and studies of the predominantly upregulated member (mouse Clca3) provide evidence for redundancy, since $\mathrm{Clca3}^{-/-}$mice still develop IL-13-dependent mucus production after viral infection and exhibit upregulation of a functional mouse Clca5 gene (14). Moreover, when we analyzed IL-13-stimulated mucus production in mouse airway epithelial cells in primary culture using more sensitive, specific, and quantitative real-time PCR assays than were used previously (20), we found induction of mouse Clca5 and newly discovered mouse $\mathrm{Clca} 7$ in addition to the predominant mouse Clca3 gene expression (Supplemental Figure 1; supplemental material available online with this article; doi:10.1172/JCI64896DS1). These findings raise the possibility that similar redundancy (and therefore difficulty in assigning function) might also occur in humans (16). However, the human CLCA gene family contains only 4 members, and, in contrast with the mouse system, we found that human CLCA1 but not CLCA2, CLCA3, or CLCA4 gene expression was increased in concert with IL-13 induction of mucous cell metaplasia in well-differentiated human airway epithelial cells, in this case using human tracheal and bronchial epithelial cells (hTECs) maintained under air-liquid interface conditions (Figure 1, A and $\mathrm{B}$ ). Levels of CLCA2 and CLCA4 were increased during cell differentiation under air-liquid interface conditions, but neither was increased by IL-13 treatment. Levels of CLCA3 mRNA were undetectable under these conditions. Moreover, whole-genome microarray analysis (and corresponding quantitative PCR [qPCR] assays) of gene expression showed marked upregulation of CLCA1 and MUC5AC mRNA levels relative to all other genes in IL-13stimulated hTECs (Supplemental Figure 2). Mucus production was signified by increased MUC5AC mRNA levels without significant changes in MUC5B gene expression (Supplemental Figure 3), consistent with a previous report (21) and indicative of other controls or cellular sources for MUC5B expression $(22,23)$. The increases in CLCA1 and MUC5AC mRNA resulted in corresponding increases in CLCA1 and MUC5AC protein levels, with significant detection of these proteins in cell lysate and apical cell supernatant (Figure 1, C and D). Confocal microscopy of IL-13stimulated hTECs immunostained for CLCA1 and MUC5AC showed colocalization in a spatial pattern of apical MUC5AC coupled with subjacent CLCA1 (Figure 1, E and F, and Supplemental Video 1). Immunostaining was specific for CLCA1 and MUC5AC since there was no staining above background in the absence of primary antibody (Supplemental Figure 3). These findings are consistent with coordinated packaging of CLCA1 and MUC5AC within mucous granules as described previously $(24,25)$ and, based on the present findings, concerted synthesis and secretion of CLCA1 and MUC5AC to the apical cell surface in response to IL-13-stimulation as well.

To define the functional role of CLCA1 in regulating mucus production, we next analyzed the effect of specific siRNA-mediated knockdown of CLCA1 gene expression. We found that stably transducing hTECs with siRNA directed against CLCA1 resulted in quantitative inhibition of IL-13-driven mucus production (Figure $1, \mathrm{G}$ and $\mathrm{H}$ ). In this system, lentiviral transfection efficiency ranged from $50 \%$ to $60 \%$ (Supplemental Figure 3) and resulted in a similar quantitative decrease in MUC5AC mRNA and protein levels. The inhibitory effect was limited to IL-13-inducible mucus production, with no significant effect on basal mucus production. In addition, the inhibitory effect was specific to CLCA1 since siRNA-mediated knockdown did not significantly affect CLCA2 or CLCA4 mRNA levels, and CLCA3 was undetectable under these conditions (Supplemental Figure 3). Together, the results indicate that CLCA1 is a master regulator of the IL-13 to mucin gene expression signaling pathway and, in contrast to the mouse system (14), appears to function without redundancy from other CLCA gene products. Thus, further studies were performed in the human system.

CLCA1-MAPK signaling in cultured cells. To determine the signaling mechanism for CLCA1-dependent induction of mucin gene expression, we next used NCI-H292 cells (derived from a lung mucoepidermoid carcinoma) to establish a cell line with stable expression of the CLCA1 gene under the control of a doxycycline-inducible promoter system (referred to herein as NCI-H292-rtTA-CLCA1 cells) (as diagrammed in Figure 2A). Analysis of NCI-H292-rtTA-CLCA1 cells indicated that overexpression of CLCA1 was sufficient for mucin gene expression based on dose-dependent induction of CLCA1 and $M U C 5 A C$ gene expression (Figure 2, B and C). As we found in primary-culture hTECs, the increases in CLCA1 and MUC5AC mRNA levels were accompanied by concomitant increases in CLCA1 and MUC5AC protein (Figure 2, D-F). The capacity of CLCA1 expression to be sufficient for mucin gene expression is consistent with our previous observations in mice, wherein gene transfer using a viral vector encoding the mouse Clca3 gene was also sufficient for mucin gene expression in vivo (14).

We next assessed downstream signaling from the CLCA1 gene product using phospho-MAPK antibody arrays to screen for any possible MAPK activation subsequent to doxycycline activation of CLCA1 expression. We observed that doxycycline treatment of NCI-H292-rtTA-CLCA1 cells caused time-dependent activation of 12 MAPKs (Figure 3A and data not shown). The largest doxycycline-induced increase in MAPK phosphorylation (doxycycline treatment compared with no treatment) was found for MAPK14 (Figure 3A), consistent with MAPK14 inhibitor blockade of cytok- 
A
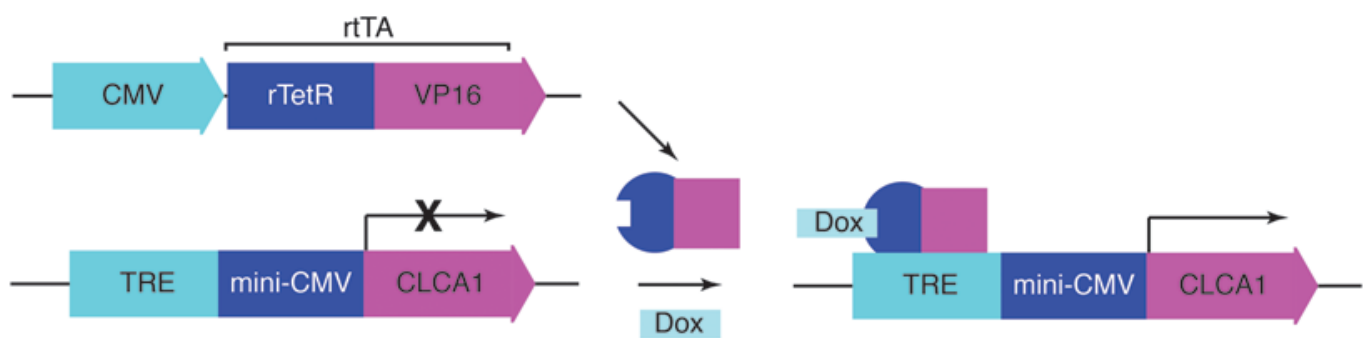

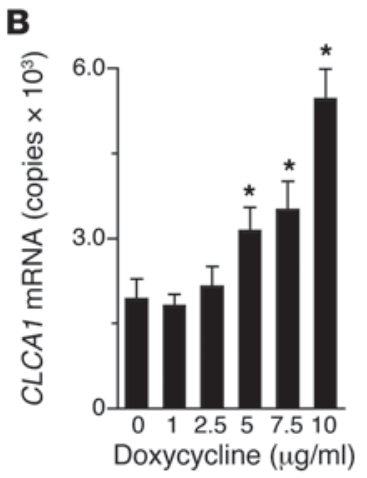

D

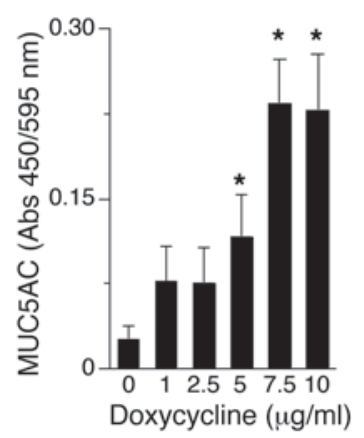

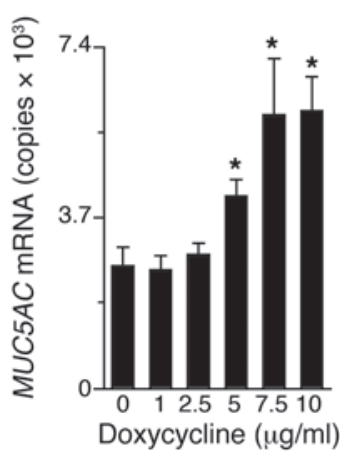

E

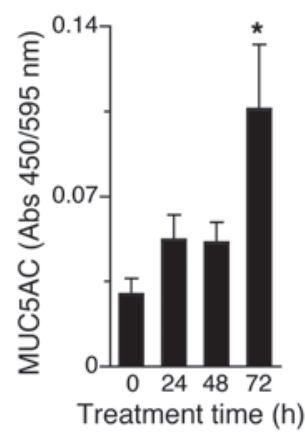

F
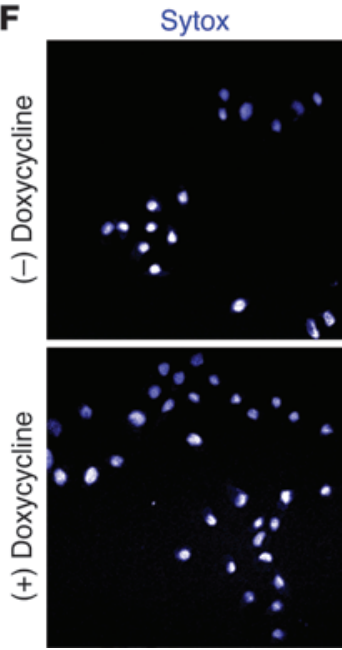

C
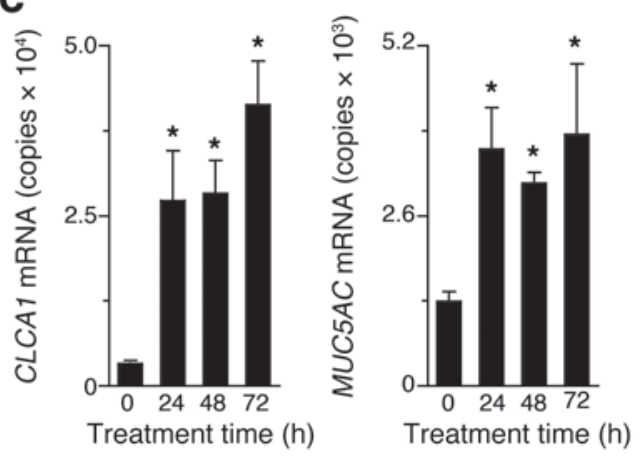
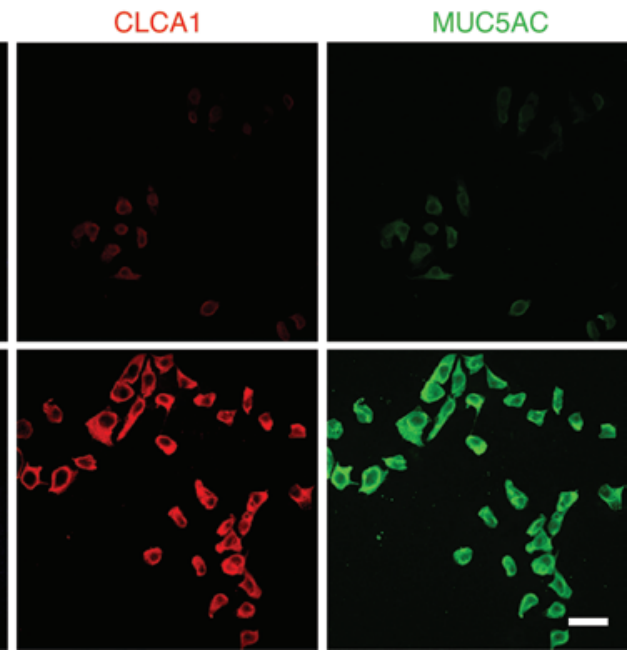

\section{Figure 2}

CLCA1 expression is sufficient for mucus production in lung epithelial cells. (A) Diagram for constructs used to establish an $\mathrm{NCl}-\mathrm{H} 292$ cell line carrying rtTA- and CLCA1-expression cassettes. (B) Doxycycline dose response for mucin gene expression in the NCI-H292-rtTA-CLCA1 cell line. Cells were treated with doxycycline $(0-10 \mu \mathrm{g} / \mathrm{ml})$ for 48 hours, and CLCA1 and MUC5AC mRNA levels were determined by real-time qPCR assay. (C) Time course for mucin gene expression in NCl-H92-rrTA-CLCA1 cells. Cells were treated with doxycycline $(7.5 \mu \mathrm{g} / \mathrm{ml})$ for 0 to $72 \mathrm{hours}$. (D) Dose response for mucus protein levels in NCl-H292-rtTA-CLCA1 cells. Cells were treated with doxycycline for 48 hours, and MUC5AC levels were determined by cell-based ELISA. (E) Time course for mucus protein levels in NCl-H92-rrTA-CLCA1 cells. Cells were treated with doxycycline ( $7.5 \mu \mathrm{g} / \mathrm{ml}$ for 0 to 72 hours), and MUC5AC levels were determined by ELISA. (F) Representative immunocytochemistry for NCI-H92-rrTA-CLCA1 cells treated with doxycycline $(7.5 \mu \mathrm{g} / \mathrm{ml}$ for 48 hours $)$ and then incubated with Sytox nuclear stain and immunostained for CLCA1 and MUC5AC. Scale bar: $100 \mu \mathrm{m}$. (B-E) Values represent mean \pm SEM. ${ }^{*} P<0.05$, increase from corresponding no doxycycline treatment control.

ine-induced MUC5AC gene expression in airway epithelial cells $(12,13,21,26)$. However, blockade was only achieved at relatively high $(10-20 \mu \mathrm{M})$ concentrations of inhibitor that may no longer be specific for MAPK14 (27). Moreover, others found no effect of MAPK14 inhibitors on PMA-induced MUC5AC expression in NCI-H292 cells or airway epithelial cells $(11,28)$. Thus, we were impressed with a similarly large increase in phosphorylation of MAPK13, which shares some degree of susceptibility to MAPK inhibitors but has no certain function in this cell system or others, especially relative to other MAPK family members. Moreover, baseline and doxycycline/CLCA1-dependent increases in MAPK activity were fully blocked with BIRB-796, which exhibits activity against the entire MAPK subfamily (i.e., MAPK11-MAPK14) (29). We therefore questioned whether MAPK13 might regulate mucin gene expression and whether BIRB-796 might be used as a tool compound for assessing this possibility. 

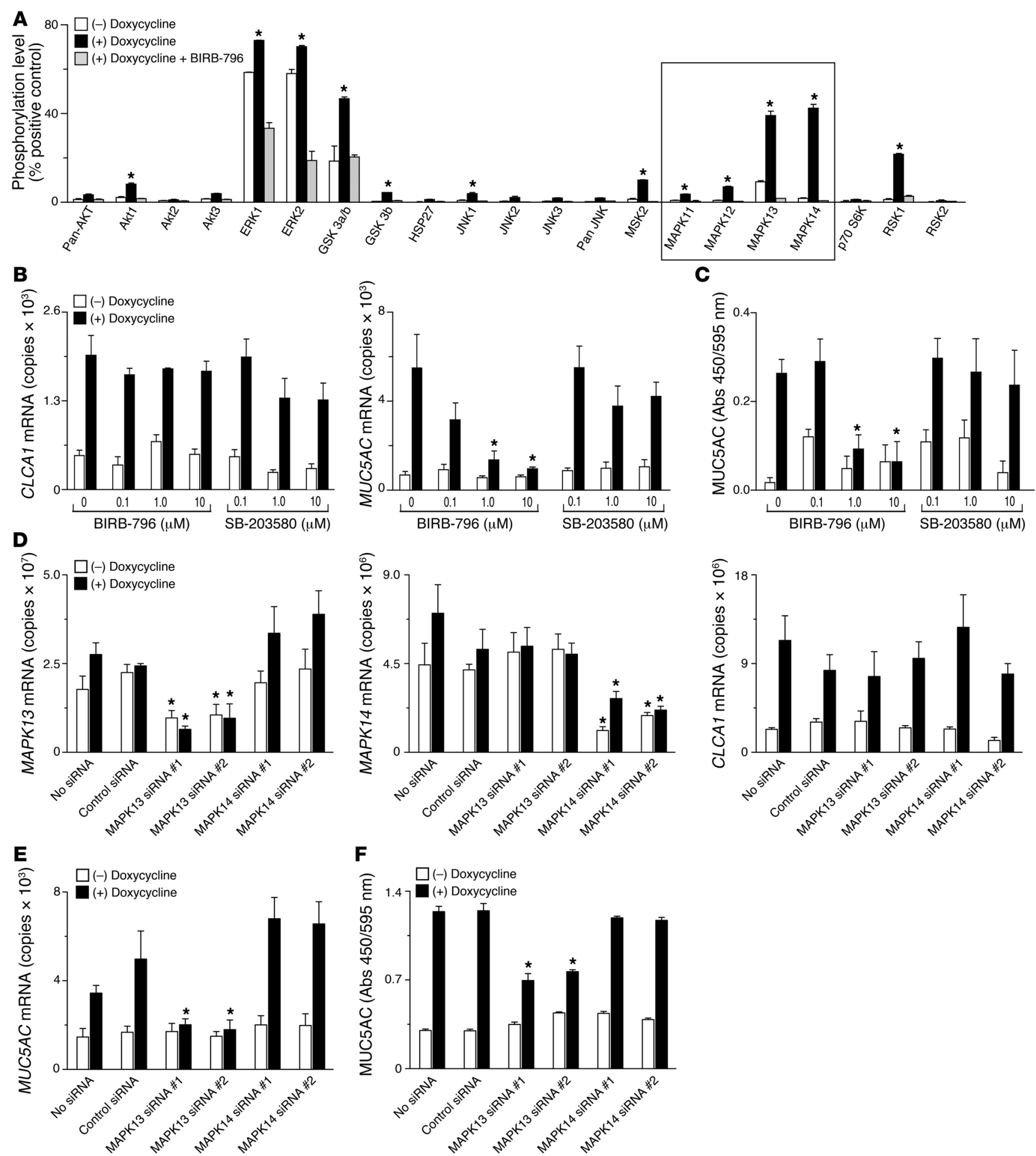

Figure 3

MAPK13 is necessary for CLCA1-driven mucus production in lung epithelial cells. (A) NCI-H292-rtTA-CLCA1 cells were treated with doxycycline ( $7.5 \mu \mathrm{g} / \mathrm{ml}$ for 18 hours) with or without BIRB-796 $(10 \mu \mathrm{M})$, and cell lysates were analyzed by phospho-MAPK antibody array. The box indicates results for MAPK values. Values represent the percentage of positive control (mean \pm SEM). ${ }^{*} P<0.05$, increase from corresponding no doxycycline control. (B) Cells were treated as in A at indicated concentrations of BIRB-796, SB-203580, and vehicle control, and levels of CLCA1 and MUC5AC mRNA were determined by real-time qPCR assay. (C) Corresponding MUC5AC levels determined by cell-based ELISA. (D) Cells were transfected with or without control or MAPK13 or MAPK14 siRNA, (25 nM) and treated with or without doxycycline (7.5 $\mu \mathrm{g} / \mathrm{ml}$ for $48 \mathrm{hours})$, and levels of MAPK13, MAPK14, and CLCA1 mRNA were determined by real-time qPCR. (E) Corresponding levels of MUC5AC mRNA for conditions in D. (F) Corresponding MUC5AC levels determined by cell-based ELISA for conditions in D. (B-F) Values represent mean \pm SEM. ${ }^{*}<<0.05$, decrease from corresponding no drug treatment or no siRNA control. 

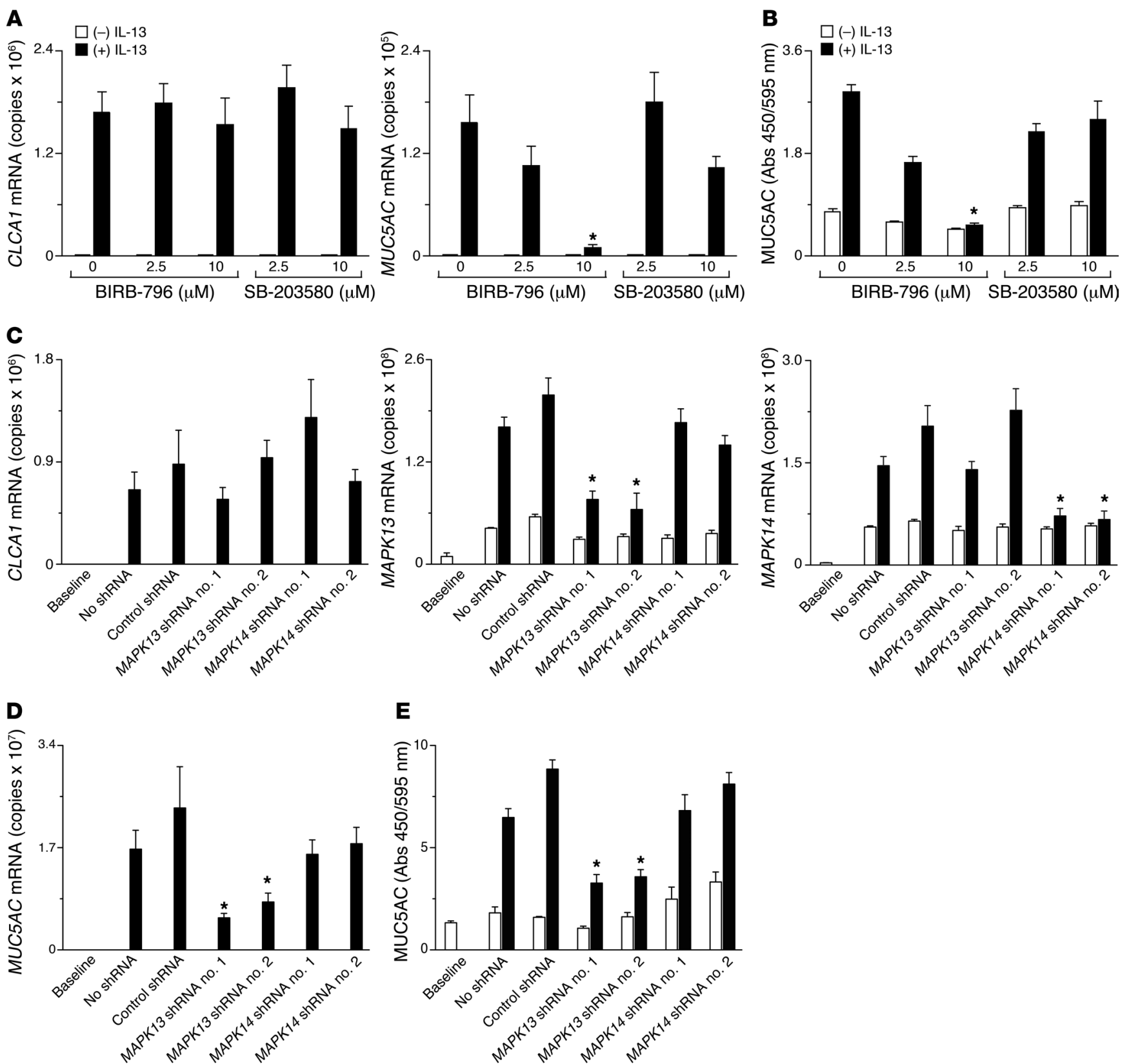

Figure 4

MAPK13 is necessary for IL-13-driven mucus production in human airway epithelial cells. (A) hTECs were incubated with IL-13 for 21 days, as described in Figure 1, with or without BIRB-796 or SB-203580, and levels of CLCA1 and MUC5AC mRNA were determined by real-time qPCR assay. (B) Corresponding MUC5AC levels determined by cell-based ELISA for conditions in A. (C) hTECs were transduced with lentivirus encoding MAPK13, MAPK14, or control shRNA and then were treated with IL-13 for 21 days, as described in Figure 1. Cell lysates were analyzed for CLCA1, MAPK13, and MAPK14 mRNA levels using real-time qPCR assay. (D) Corresponding levels of MUC5AC mRNA. (E) Corresponding MUC5AC levels determined by cell-based ELISA. (A-E) Values represent mean \pm SEM. ${ }^{*} P<0.05$, decrease from corresponding no drug treatment or shRNA control condition.

We subsequently found that BIRB-796 markedly inhibited doxycycline-induced MUC5AC mRNA and MUC5AC protein production, whereas SB-203580, which more selectively blocks MAPK14 (30), did not significantly inhibit MUC5AC synthesis (Figure 3, B and C). We found no effect of MAPK inhibition on CLCA1 expression. We next used MAPK13 RNAi to suppress expression and thereby define loss of function for MAPK13 in the regulation of mucin gene expression. We found that suppression of MAPK13 expression did not influence doxycycline-induced expression of CLCA1 but completely blocked expression of MUC5AC gene expression (Figure 3, D and E). By contrast, treatment with MAPK14 RNAi did not block doxycycline-induced MUC5AC synthesis or influence CLCA1 mRNA levels (Figure 3, $\mathrm{D}$ and $\mathrm{E})$. Blockade of MUC5AC mRNA induction was reflected 


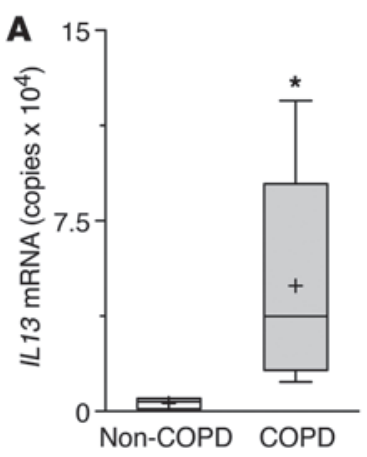

B

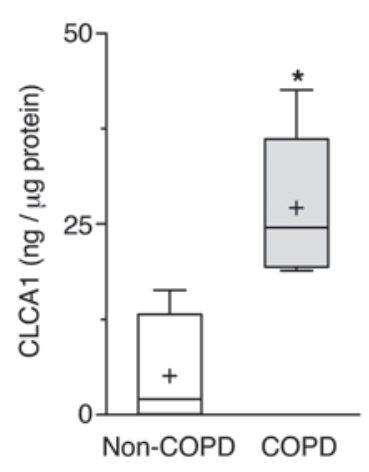

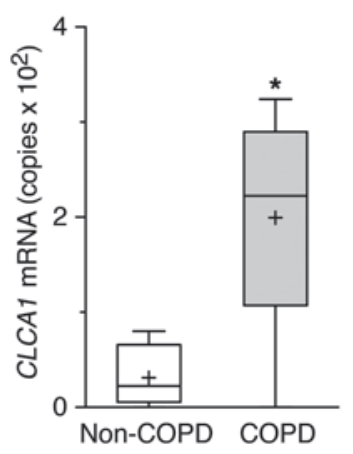
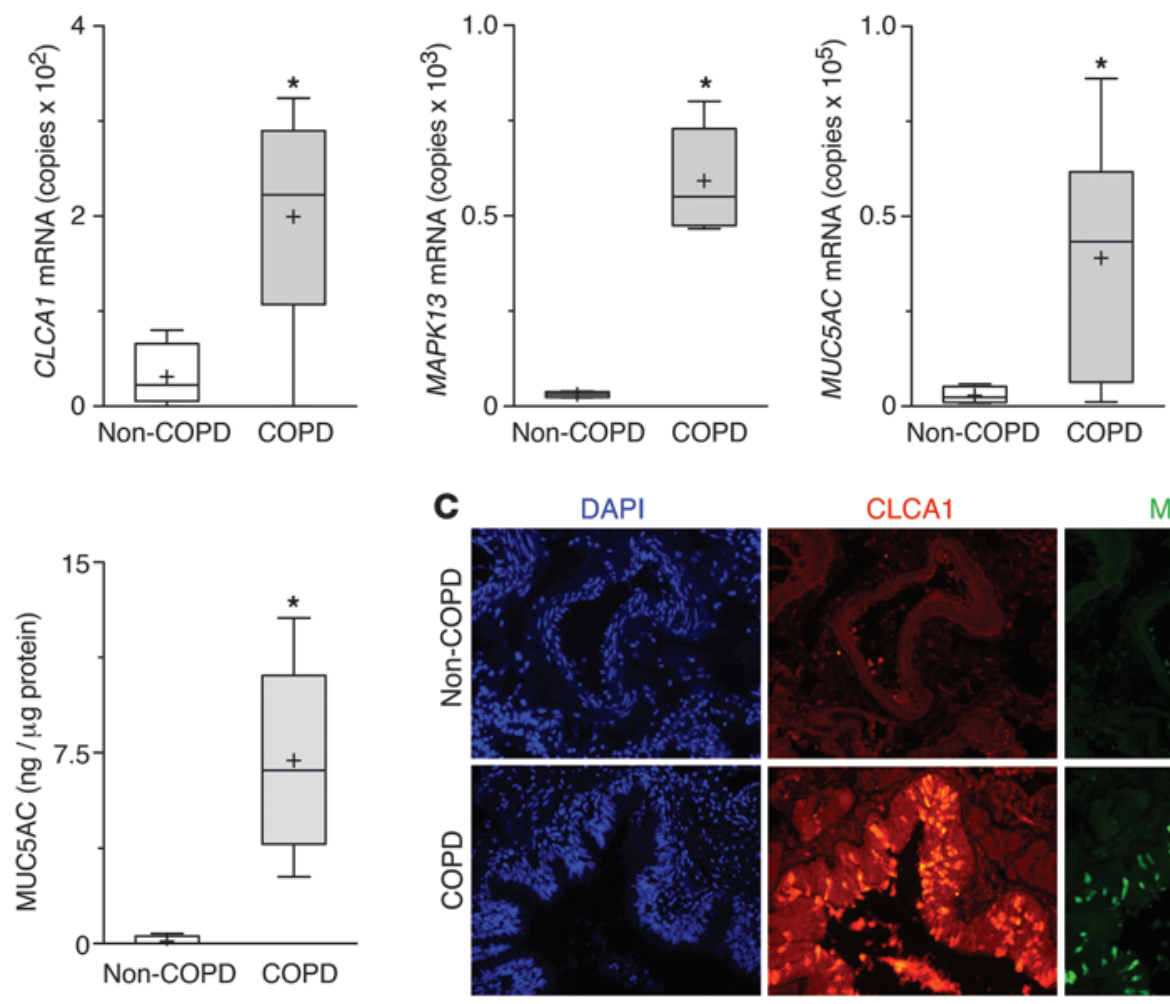

CLCA1

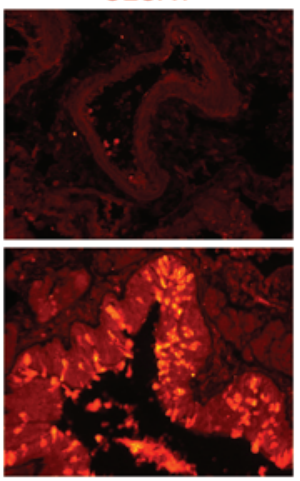

MUC5AC

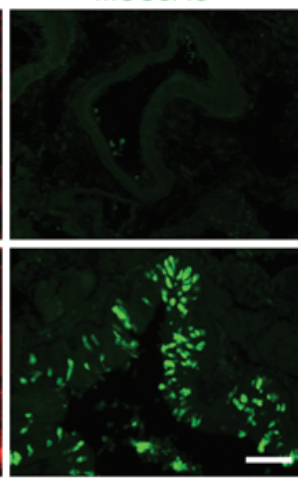

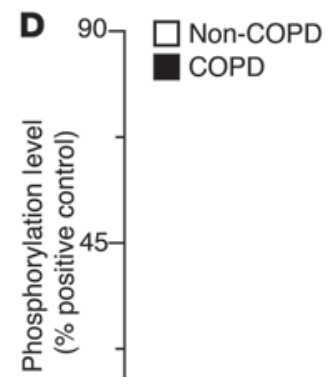

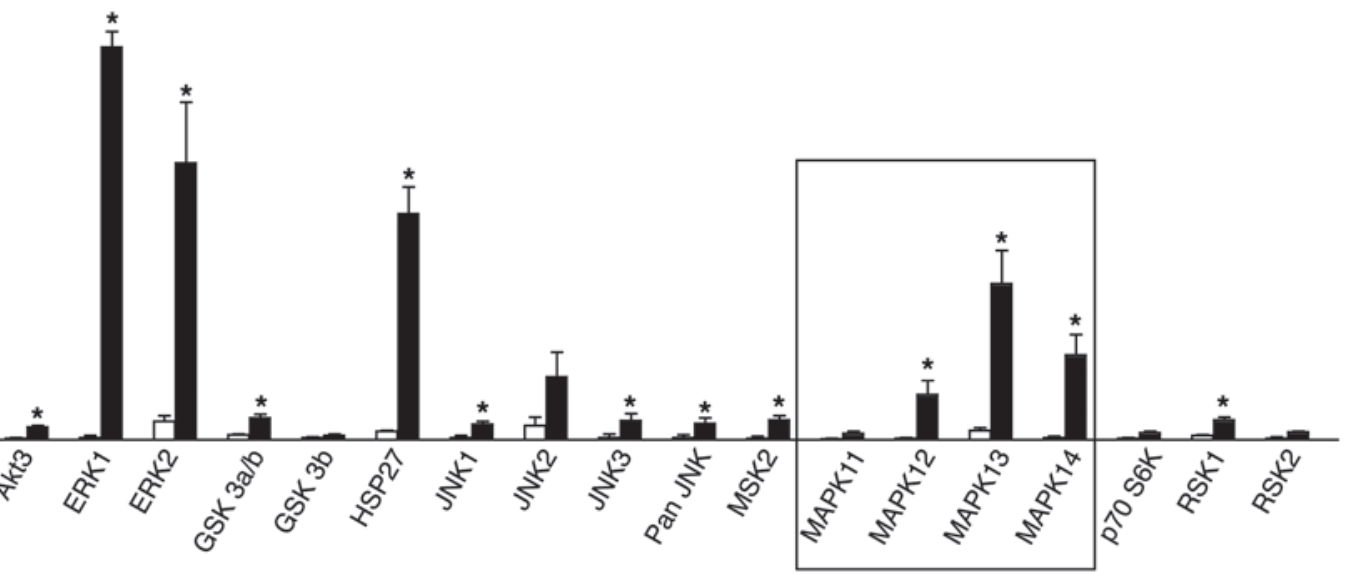

Figure 5

Evidence of a CLCA1 to MAPK13 signaling pathway for IL-13-stimulated mucus production in COPD. (A) Levels of IL13, CLCA1, MAPK13, and MUC5AC mRNA in lung tissues from lung transplant donors without COPD $(n=4)$ and recipients with very severe COPD $(n=9)$. (B) Levels of CLCA1 and MUC5AC determined by ELISA from lung samples obtained as in A. Values represent mean of triplicate values for each subject. (A and B) For box-and-whisker plots, whiskers represent range, the plus sign indicates mean values, and boxes indicate 25th to 75 th percentiles. ${ }^{*} P<0.05$, difference from non-COPD control. (C) Representative photomicrographs of corresponding lung sections subjected to immunostaining for CLCA1 and MUC5AC. Scale bar: $100 \mu \mathrm{m}$. (D) Levels of MAPK activation based on analysis of phospho-MAPK antibody array for lung samples obtained as in A. Values represent percentage of positive control (mean $\pm \mathrm{SEM}$ ). ${ }^{*} P<0.05$.

in inhibition of corresponding MUC5AC protein levels (Figure $3 \mathrm{~F})$. These results placed MAPK13 downstream of CLCA1 and upstream of MUC5AC gene expression in NCI-H292 cells.

A similar influence of MAPK13 over mucin gene expression was found in primary-culture hTECs incubated with IL-13. Thus, p38-MAPK blockade with BIRB-796 markedly suppressed IL-13induced MUC5AC gene expression, without affecting correspond- ing CLCA1 mRNA levels, whereas treatment with SB-203580 had no significant effect on CLCA1 or MUC5AC gene expression (Figure 4, $A$ and B). Furthermore, suppression of MAPK13 expression (using 2 different shRNA sequences delivered with a lentiviral vector) did not influence IL-13-induced expression of CLCA1 but significantly blocked the expected increase in MUC5AC mRNA levels (Figure 4, C and D). In this system, lentiviral transfection efficiencies of $50 \%$ 
A BIRB-796-MAPK13 model

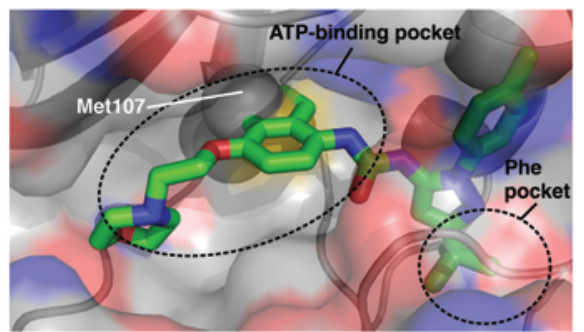

D Compound 61-MAPK13
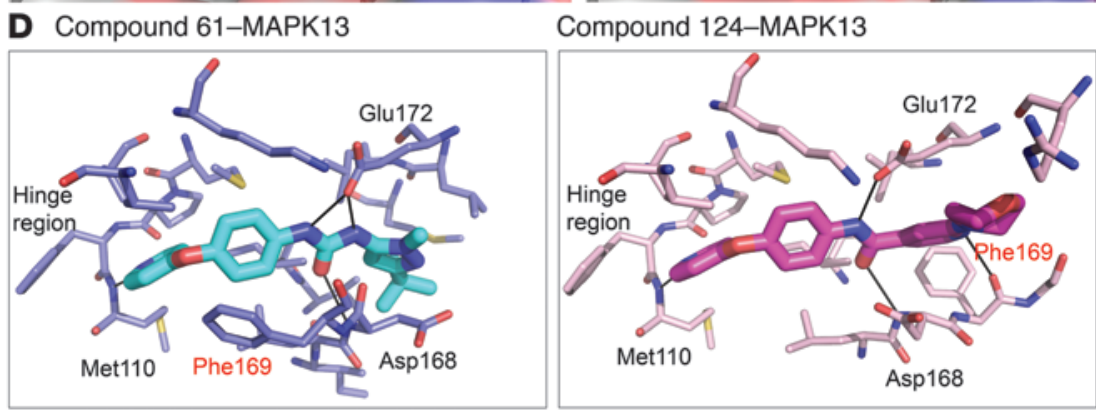

B Compound 61-MAPK13

$\mathbf{F}$

C Compound 124-MAPK13

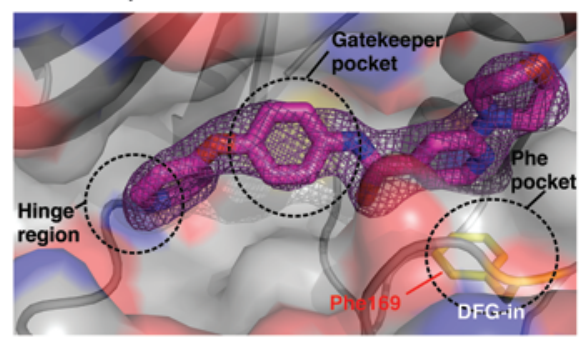

E
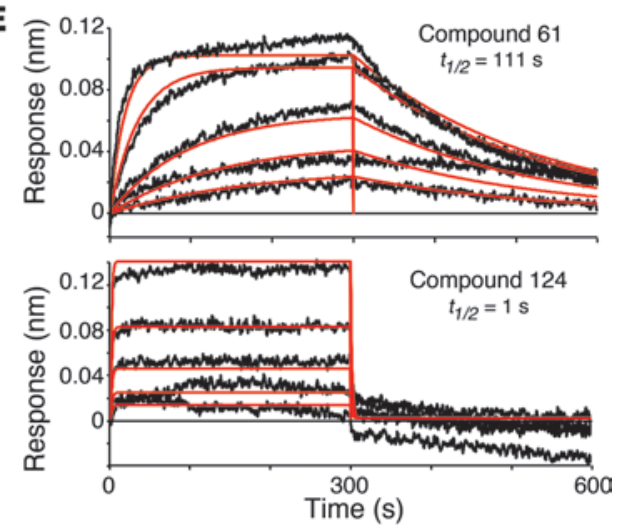

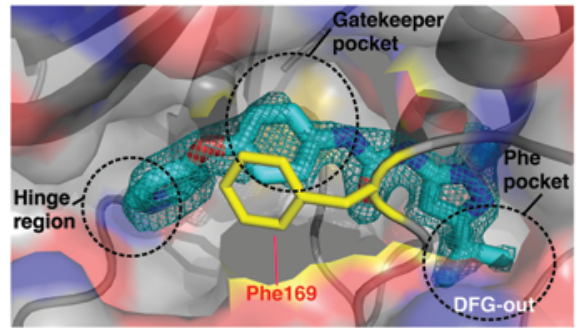

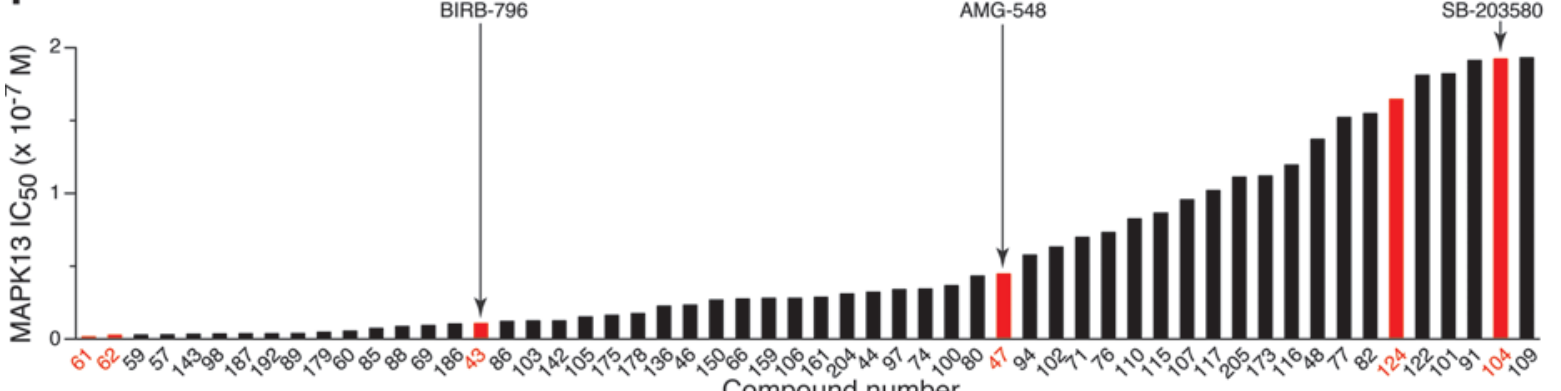

Compound number
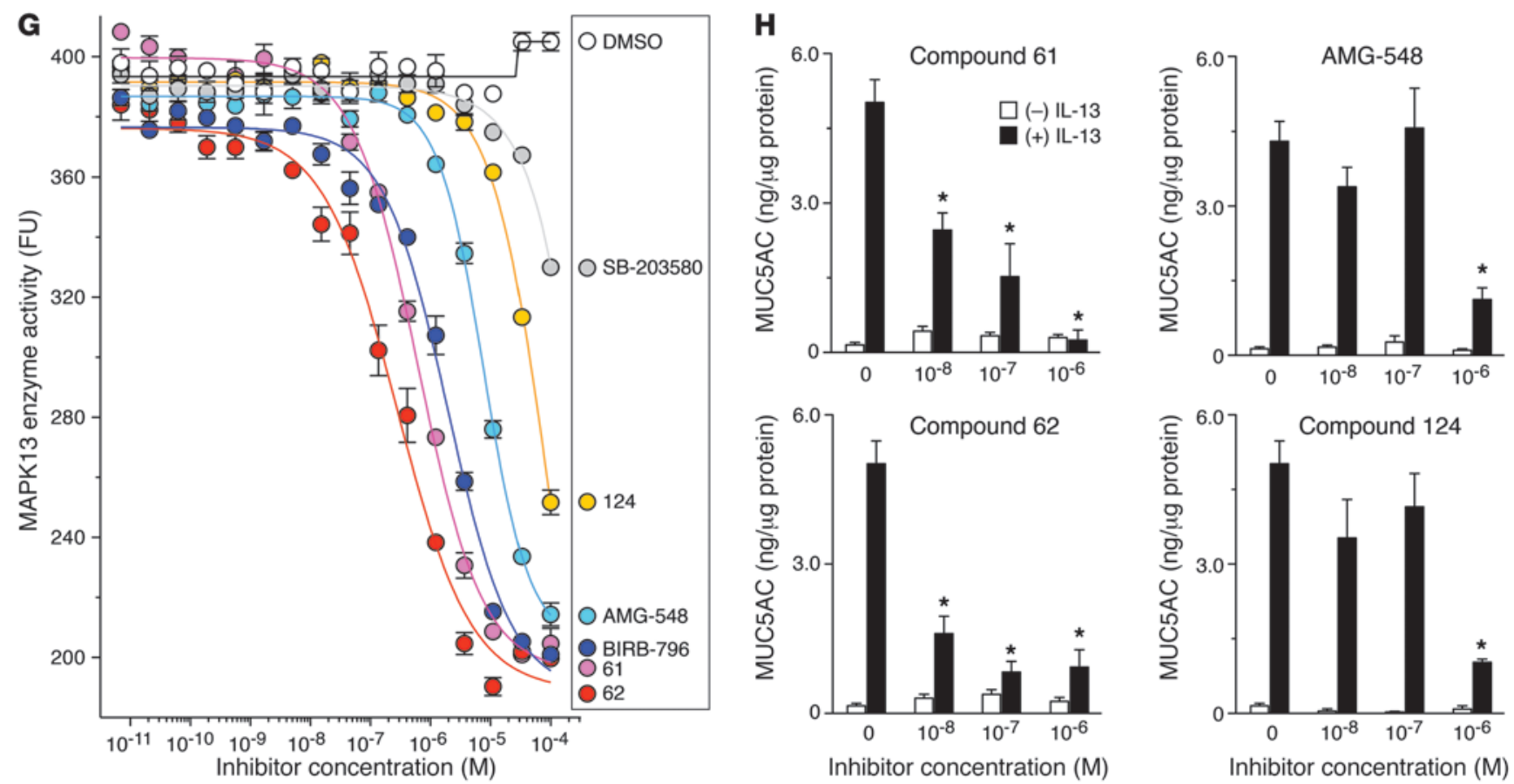

Inhibitor concentration (M) 


\begin{abstract}
Figure 6
Discovery and validation of potent MAPK13 inhibitors. (A) Superposition of BIRB-796 into the MAPK13 binding pocket. The position of the gatekeeper Met107 is shown in a space-filling model. Circles indicate ATP-binding and Phe pockets. (B) Crystal structure of compound 61 (cyan) bound to MAPK13 in DFG-out mode. Difference electron density for the compound following rigid-body refinement is shown in mesh. Circles indicate binding pocket features. Phe169 (of the DFG sequence) is highlighted in yellow. (C) Crystal structure of compound 124 (magenta) bound to MAPK13 in DFG-in mode. (D) MAPK13 binding pockets for compound 61 (cyan) and compound 124 (magenta). All residues that make van der Waals contacts or polar contacts are shown. Residues that make direct hydrogen bonds (black lines) with inhibitors are labeled in black. (E) BioLayer interferometry sensorgrams for compound 61 and 124 binding to MAPK13. Processed data are shown as black lines with global kinetic fits overlaid as red lines. (F) MAPK13 inhibitory activity in a screen of test compounds (numbered from 43-205 and arranged from most to least potent) using IMAP assay. Values represent $I C_{50}$ for compounds with values $<20 \mu \mathrm{M}$. Redcolored bars indicate compounds selected for detailed analysis. (G) Concentration response for MAPK13 inhibition for indicated test compounds. Values represent fluorescence polarization $(\mathrm{mP})$ from IMAP assay. (H) hTECs were cultured with or without IL-13 for 21 days with the indicated compounds, and MUC5AC levels were determined by ELISA. ( $\mathbf{G}$ and $\mathbf{H}$ ) Values represent mean \pm SEM. ${ }^{*} P<0.05$, decrease from no drug treatment condition.
\end{abstract}

to $60 \%$ again resulted in a similar quantitative decrease in MAPK13 and MUC5AC mRNA and protein levels. Transduction of airway epithelial cells with lentivirus encoding MAPK14 shRNA achieved a similar selective decrease in target $\mathrm{mRNA}$ but showed no significant effect on IL-13-induced MUC5AC gene expression (Figure 4D) or CLCA1 mRNA levels (Figure 4C). Blockade of MUC5AC mRNA induction was again reflected in significant decreases in corresponding MUC5AC protein levels (Figure 4E).

CLCA1/MAPK13 signaling in buman lungs. We next extended our studies of CLCA1/MAPK13 signaling in isolated cells to studies of this pathway in whole lungs with excess mucus production. Our approach took advantage of whole lung explants from recipients undergoing lung transplantation for very severe (GOLD stage IV) COPD compared with lungs from non-COPD donors. Limited clinical information was available for non-COPD subjects, but the group of patients with very severe COPD was characterized by severe emphysema and symptoms of chronic bronchitis signified by excess mucus production (Supplemental Table 1). To address the relationship of CLCA1 expression to airway mucus production, we examined lung tissue core samples directed to airways based on computed tomography imaging (31). In these airway-rich core samples, we found a significant increase in CLCA1 and MUC5AC mRNA as well CLCA1 and MUC5AC protein levels in subjects with COPD compared with subjects without COPD (Supplemental Figure 4). Moreover, CLCA1 levels correlated with MUC5AC levels across the group of COPD and non-COPD lung samples (Supplemental Figure 4).

We next performed a more detailed analysis of the CLCA1/ MAPK13 signaling pathway in a subset of subjects and found that lung tissue obtained from patients with COPD exhibited significant increases in IL13 and MUC5AC gene expression (consistent with our previous observations in a smaller group of subjects; refs. 6,8 ) as well as increased CLCA1 and MAPK13 gene expression (Figure 5A). The increases in CLCA1 and MUC5AC mRNA were also reflected in increased levels of the corresponding proteins (Fig- ure 5B). Immunostaining of lung tissue samples demonstrated colocalization of CLCA1 and MUC5AC in mucosal mucous cells (Figure 5C). Furthermore, increases in CLCA1 and MAPK13 gene expression were accompanied by significant increases in MAPK13 activation that were relatively prominent compared with those of other MAPKs (Figure 5D). Thus, IL-13 stimulation of a CLCA1/ MAPK13 signaling pathway to mucin genes found in human airway epithelial cells in culture appears to also be activated in human lungs in vivo during chronic obstructive lung disease.

We also investigated whether the homologous Clca/Mapk signaling pathway could be found in whole lungs of mice. However, we recognized that the observed differences between the mouse Clca and human CLCA gene loci and the apparent redundancy in mouse Clca responsiveness to IL-13 signaling might indicate a corresponding difference in downstream human CLCA versus mouse Clca signaling as well. Nonetheless, the availability of Mapk13deficient mice (32) provided a suitable genetic approach for testing whether a comparable signaling system might be present in a potentially useful animal model. In fact, similar to that in the human system, we found an increase in mouse Clca3 mRNA levels in response to IL-13 administration in the lungs of Mapk13-1- mice and same-strain wild-type control mice. However, we found no difference in levels of Muc5ac mRNA in the lung or corresponding Muc5ac immunostaining in the airways of Mapk13-1- mice compared with those of wild-type mice (Supplemental Figure 5). Similarly, we found the usual increase in mouse Clca3 but no difference in $M u c 5 a c$ mRNA response to IL-13 in primary-culture mouse tracheal epithelial cells from Mapk13-/- and wild-type control mice (Supplemental Figure 5). Together, these findings reinforced the position that the mouse model was not representative of the human for IL-13-dependent control of airway mucus production and that further studies to inhibit excess mucus production might be best performed in humans or human subject materials.

Design and validation of MAPK13 inbibitors. Taken together, our results in human cells and lungs pointed to a central role for MAPK13 in regulating airway mucus production and therefore suggested the utility of a more potent MAPK13 inhibitor. To develop such a compound, we used a drug discovery strategy known as scaffold hopping that starts with a known active compound and ends with a novel chemical entity by modifying the central core structure of the molecule $(33,34)$. In the present case, the strategy was designed to take advantage of MAPK13 and MAPK14 homology (60\% sequence identity) and thereby provide new analogs of MAPK14 inhibitors with improved affinity for MAPK13. We recognized that BIRB-796 exhibited potent inhibition of MAPK14 (the original target for this compound) but relatively weak activity for MAPK13 (29). The selectivity of BIRB-796 for MAPK14 is based largely on incorporating a naphthalene group into part of the molecule (i.e., a naphthalene moiety) that can readily occupy a distinct binding site (designated the gatekeeper pocket) in MAPK14 but not the more confined pocket in MAPK13. The difference in access to this pocket appeared to be under the control of the adjacent gatekeeper residue, which is a small threonine in MAPK14 compared with a large methionine in MAPK13. To initiate our structure-based drug design approach, we first crystallized and determined the structure of inactive uncomplexed MAPK13 at high resolution. Indeed, superposition of the MAPK14-BIRB-796 cocrystal structure onto MAPK13 revealed interference (i.e., a steric clash) between the gatekeeper methionine and the inhibitor naphthalene moiety (Figure 6A and Supplemental Figure 6). 


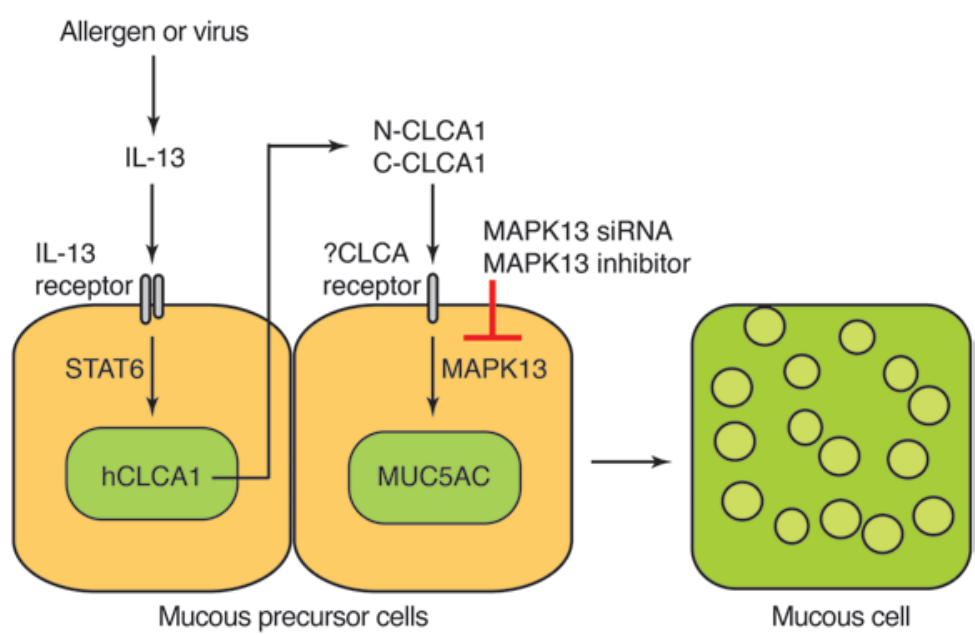

\section{Figure 7}

Scheme for CLCA1/MAPK13 signaling to mucus production. The CLCA1/MAPK13 signaling pathway is initiated when environmental stimuli (e.g., allergen or virus) stimulate IL-13 production and subsequent activation of IL-13 receptor on the surface of mucous precursor cells. IL-13 receptor activation leads to STAT6 activation and consequent induction of CLCA1 gene expression. The CLCA1 protein is secreted as $\mathrm{N}$-terminal and $\mathrm{C}$-terminal CLCA1 (N-CLCA1 and C-CLCA1) peptide fragments that might activate a putative CLCA1 receptor and in turn MAPK13 to drive a consequent increase in mucin (especially MUC5AC) gene expression and mucus production in airway mucous cells.
We therefore used BIRB-796 as a starting compound to build slimmer analogs that would no longer clash with the gatekeeper residue and maintain other beneficial structural features to thereby exhibit greater potency for MAPK13. We were particularly interested in BIRB-796 because it binds to MAPK14 in the Asp-Phe-Glyout mode (DFG-out mode) (35). This binding mechanism results in rotation of the conserved DFG motif out of the Phe-binding pocket (i.e., DFG-out) and thereby produces a prolonged dissociation rate that might offer a pharmacological advantage (35-37). With the aid of protein structure modeling, we designed and synthesized analogs that incorporated a smaller monoaryl ring in place of the bulky naphthalene in BIRB-796 to prevent clashing with the gatekeeper methionine (Supplemental Figure 7). Analysis of analog-MAPK13 cocrystals confirmed successful binding that preserved contacts and hydrogen bonds with the same MAPK13 residues (Figure 6, B and C; Supplemental Figure 8; and Supplemental Table 2). However, while some analogs (e.g., compound 61) demonstrated the expected DFG-out binding mode, other analogs (e.g., compound 124) adopted DFG-in binding due to conformational constraints imposed by the fit to the binding pocket. In concert with these results, analogs with DFG-out binding (e.g., compound 61) maintained slow dissociation kinetics, while those exhibiting DFG-in binding (e.g., compound 124) formed short-lived complexes (Figure 6, D and E). We anticipated that these structural and kinetic differences might translate into differences in compound potencies in enzyme and cellular assays of MAPK13 activity.

All analogs were tested for inhibition of MAPK13 enzyme activity using an immobilized metal affinity polarization (IMAP) assay that detected fluorescence polarization as a function of remaining MAPK13 activity. As expected, DFG-out binding compounds (e.g., compound 61 and, a similar compound, compound 62) displayed highly potent MAPK13 inhibition, whereas DFG-in binding compounds (e.g., compound 124) with otherwise similar structure displayed 65 -fold to 120 -fold lower inhibitory activity (Figure 6F). Structurally distinct AMG-548 and SB-203580 also exhibited weak inhibitory activity consistent with lower affinity for MAPK13 compared with MAPK14 $(30,38)$. More detailed 16-point inhibitor concentration-response curves were performed for representative compounds (Figure 6G). This analysis further confirmed that, within our chemical series, the most active DFG-out compounds (i.e., compounds 61 and 62) were more potent than a representa- tive DFG-in compound (i.e., compound 124) and compared favorably with less selective inhibitors designed to inhibit MAPK14, i.e., AMG-548 and SB-203580 (Figure 6G and Supplemental Table 3). As expected, all of these compounds continued to show inhibitory activity against MAPK14 as well (Supplemental Table 4 and Supplemental Figure 9), since the compounds should continue to fit into the MAPK14 gatekeeper pocket and no changes were made to interfere with binding at other sites in the MAPK molecules.

Nonetheless, the greater potency of newly designed compounds for blocking MAPK13 suggested that these compounds would have greater potency for blocking IL-13-dependent mucus production. Accordingly, we next tested the effect of representative analogs on mucus production in comparison to the effects of compounds designed to inhibit MAPK14. We found that potency for MAPK13 inhibition correlated closely with blockade of mucus production. Thus, the most potent MAPK13 inhibitors (compounds 61 and 62) suppressed IL-13-induced MUC5AC production in primaryculture human airway epithelial cells with $50 \%$ inhibition at $10^{-8}$ $\mathrm{M}$, whereas treatment with less potent MAPK13 inhibitors (compound 124 and AMG-548) had no significant effect on MUC5AC level until treatment with $10^{-6} \mathrm{M}$ (Figure $6 \mathrm{H}$ ). None of the tested compounds resulted in significant changes in transepithelial electrical resistance, even at $10^{-6} \mathrm{M}$, indicating a lack of cytotoxic effect despite treatment for 21 days (Supplemental Figure 10). Taken together, the findings reveal a potent and effective action of rationally designed MAPK13 inhibitors in blocking IL-13-induced mucus production in primary-culture human airway epithelial cells under culture conditions that maintain the features of a well-differentiated airway mucosa found in vivo.

\section{Discussion}

In this report, we establish the critical role of CLCA1 and MAPK13 in controlling excess mucus production that is typical of inflammatory airway disease. We use genetic and chemical tools to define a signaling pathway that begins with IL-13 induction of CLCA1 gene expression and proceeds to CLCA1-mediated activation of MAPK13 and consequent MUC5AC mucin gene expression (Figure 7). Pathway function was established by gain and loss of function for CLCA1 and MAPK13 in primary-culture human airway epithelial cells and representative cell lines and in lung explants from patients with excess mucus due to COPD. This approach to 
incorporate human subjects and materials was essential due to the substantial differences in CLCA protein families between humans and other species (including mice) $(14,16)$ and the consequence divergence in downstream CLCA signaling as well.

Indeed, the first reason for the present advance in understanding CLCA1 function was the shift from mouse to human model systems. Thus, the initial discovery of the role of CLCA proteins in airway disease was derived from studies of inbred and intercrossed mice in which whole-genome assessment revealed linkage between mouse Clca3 gene expression and mucus production after respiratory viral infection (14). However, this model also showed that loss of Clca3 function (using newly developed $\mathrm{Clca3}^{-/-}$mice) did not influence IL-13-driven mucus production and led to the initial concern that $\mathrm{Clca}$ proteins were not involved in regulating excess mucus production during inflammatory airway disease. However, further genetic analysis also revealed that the mouse Clca gene family contained at least 7 members, so that gene duplication and functional redundancy could account for preservation of the postviral mucus phenotype if only a single Clca gene was inactivated. In fact, additional analysis of this mouse model showed that other Clca family members were also sufficient for driving mucus production $(14,15)$. Similarly, in this study, we found IL-13 upregulation of mouse Clca5 and Clca7 as well as predominant Clca3 gene expression in mouse airway epithelial cells. In addition, we found that the connection between CLCA expression and MAPK13 activation present in humans was not apparent in mice. Thus, IL-13 induction of Muc5ac gene expression was not blocked by Mapk13 deficiency (using Mapk13-/- mice compared with wild-type control mice) either in vivo (using mice treated with inhaled IL-13) or in vitro (using primary-culture mouse tracheal epithelial cells treated with IL-13). It was therefore critical to assess the epithelial mucus system in humans to establish selective induction of CLCA1 and activation of MAPK13 as key events for regulating IL-13-inducible mucus production and secretion in isolated cells and whole lungs.

A second reason for the current progress on CLCA1 function relates to the role of CLCA1 as a signaling molecule compared with the original assignment (based on sequence homology) as an integral membrane protein that functioned as a calcium-activated chloride channel (CaCC). In that regard, even the initial bioinformatic (16) and experimental data $(17,18,39)$ indicated that CLCA proteins generally lack the structural features to form ion channels by themselves, since they either contain only a single transmembrane anchor or exhibit secretion from the cell in soluble form. Moreover, subsequent findings were more consistent with the proposal that CLCA1 effects on chloride transport may be based on increasing conductance of endogenous CaCCs (40). It further appears (based on analysis of crude membrane preparations) that secreted CLCA proteins are first processed intracellularly into $\mathrm{N}$-terminal and C-terminal peptide fragments (18, 39, 41-44). The present findings support this alternative proposal as well, in that CLCA1 is colocalized within the cell along with MUC5AC in secretory granules and is secreted to the apical cell surface in response to IL-13. Our initial structural work with purified human CLCA1 protein suggests that a novel form of self-cleavage is required for secretion and that the consequent production of the $\mathrm{N}$-terminal fragment of CLCA1 is sufficient for stimulating CaCC activity (45). The present connection between CLCA1 and MAPK13 activation and downstream mucin gene expression further support the proposal that CLCA1 functions as a signaling molecule, since MAPKs in general and p38-MAPKs in particular have always been acknowledged to transmit extracellular signals to intracellular targets via upstream receptors $(46,47)$. The nature of a putative CLCA1 receptor that might be linked to MAPK13 activation is currently under study. Similarly, at least some CaCCs may also trigger MAPK signaling (e.g., TMEM16A stimulates ERK1/2 activation) (48), but the CaCC signaling pathway to activate chloride transport still needs to be defined. Nonetheless, the combination of previous and present work serves to position CLCA1 as a central signaling protein for controlling both the glycoprotein and fluid components of mucus production.

In regard to CLCA1 control of mucus production, a third reason for the present advance was the need for an unbiased approach to CLCA1 signaling targets and the particular advantage of antibody arrays to screen for these targets to find MAPK13. The relatively unstudied MAPK13 was originally described as responsive to inflammatory cytokines and cellular stress but downstream targets were uncertain (49). Subsequent reports suggested a role for MAPK13 in keratinocyte differentiation and survival $(50,51)$, microtubule assembly and neurogeneration (52), attenuation of insulin secretion (53), and cell proliferation in skin cancer and cholangiocarcinoma $(54,55)$; however, no complete scheme has emerged for MAPK13 activity in human disease. Instead, most previous work has concentrated on other MAPK family members, primarily MAPK14, as contributing to inflammation in the airway and other sites (56) as well as driving mucus production (12, 26). Based on these observations, drug development activity was generally directed at MAPK14, yielding inhibitors with increased kinase selectivity and antiinflammatory activity $(19,57)$. The present results assign a new function for MAPK13 and should turn the field to a different strategy for the study of MAPK biology and blockade. Though here again we caution an approach that uses the mouse model, in which MAPK13 has recently been shown to regulate other types of kinase-dependent responses (e.g., TLR4-driven ERK1/2 activation in immune cells) (58).

Finally, a key reason for the lack of previous progress on MAPK13 function was the absence of potent chemical inhibitors of MAPK13. Indeed, this project benefited greatly from the identification of more potent MAPK13 inhibitors. We developed these inhibitors guided by structural analysis of MAPK13-inhibitor cocrystals and scaffold hopping from compounds that were designed previously to bind selectively to the homologous MAPK14. Our approach allowed for the discovery of potent MAPK13 inhibitors that better fit the MAPK13 gatekeeper pocket and maintained a DFG-out mode with slow dissociation kinetics $(35,36)$. Compounds that achieved these end points (e.g., compounds 61 and 62) provided for an approximate 100 -fold increase in MAPK13-inhibitor complex half-life and a 100-fold increase in mucus inhibition. These properties should translate into more sustained and potent effects for use as tool compounds and for further drug development. It is also possible that the combination of gatekeeper- and Phe-pocket binding will improve kinase selectivity. However, the present inhibitors maintain their activity against MAPK14. Thus, the chemical approach to MAPK13 inhibition was scientifically complemented and validated by the concomitant genetic approach, and additional work will have to be done to fully characterize the kinase specificity of the present compounds and perhaps to develop others with greater specificity. Recent success in developing extremely selective MAPK14 inhibitors $(59,60)$ suggests that this is an achievable step. Although compounds with combined MAPK13 and MAPK14 activity may 
offer a clinical advantage for diseases such as COPD that manifest both mucus and inflammatory components in the disease process. Indeed, there is ongoing clinical development of MAPK14 inhibitors to block inflammation in COPD and asthma $(61,62)$. In either case, the present identification and validation of the CLCA1 to MAPK13 signaling pathway to excess mucus production offers an opportunity to better define the regulation of a major disease end point and to respond to the unmet need for an anti-mucus therapeutic for inflammatory airway disease.

\section{Methods}

See the Supplemental Methods for more details.

Primary culture of human airway epithelial cells. hTECs were isolated by enzymatic digestion, seeded onto permeable filter supports, and grown as described previously (6). For the present experiments, cells were cultured in DMEM-Ham's F12 medium with 2\% NuSerum (BD), Primocin (100 $\mu \mathrm{g} /$ $\mathrm{ml}$, InvivoGen), and retinoic acid $\left(1 \times 10^{-8} \mathrm{M}\right.$, Sigma-Aldrich) with or without IL-13 (50 ng/ml, Peprotech) under submerged conditions for 2 days and then air-liquid interface conditions for 3 weeks. Cells were also cultured in the presence or absence of inhibitors that were added 1 day before addition of IL-13 and were readded with each IL-13 treatment (twice per week). Transepithelial electrical resistance of cell cultures was monitored as described previously (63).

Real-time $q P C R$ assay. Target mRNA levels were quantified with real-time qPCR using fluorogenic probe/primer combinations specific for CLCA1, CLCA2, CLCA4, MUC5AC, MAPK13, MAPK14, and GAPDH (Supplemental Table 5). All PCR assays were quantitative and used plasmids containing the target gene sequences as standards. All probes were designed to span an intron and did not react with genomic DNA. All real-time PCR data were normalized to the level of GAPDH $\left(1 \times 10^{7}\right.$ copies $)$ to correct for variation between samples as described previously (8).

Immunostaining. Cells were fixed, permeabilized, treated for epitope retrieval, and then were incubated with mouse anti-MUC5AC biotin-conjugated $\mathrm{mAb}$ and rabbit anti-CLCA1 antibody followed by Alexa Fluor 488or 555-conjugated streptavidin or Alexa Fluor 555- or 633-conjugated goat anti-rabbit secondary antibody, counterstained with DAPI or Sytox Green and then imaged by confocal or conventional microscopy. Lung sections were incubated with citrate-based Antigen Unmasking Solution (Vector Laboratories) for antigen retrieval and then with anti-MUC5AC $\mathrm{mAb}$ and anti-CLCA1 antibody, followed by Alexa Fluor 488-conjugated streptavidin and Alexa Fluor 555-conjugated goat anti-rabbit IgG.

Generation of shRNA-expressing cells. Lentiviral vectors expressing siRNA from shRNA were obtained from the MISSION shRNA library (Sigma-Aldrich). A set of shRNA clones (constructed within the lentivirus plasmid pLKO.1-Puro) was obtained for each of the following mRNA targets: CLCA1 (NM_001285), MAPK13 (NM_002754), and MAPK14 (NM_001315). Lentiviral particles for each of the clones were generated by transfecting HEK 293 T cells with shRNAi-pLKO.1-Puro plasmid, pHR'8.2deltaR packaging plasmid, and PCMV-VSV-G envelope plasmid using Fugene6 (Roche Applied Science) as the transfection agent (64). The shRNA lentiviral particles were added to the apical and basal sides of hTEC cultures for 24 hours at MOI 1, and 10 days later, cells were treated with or without IL13 as described above.

MUC5AC ELISA. For cell-based MUC5AC ELISA, cells were fixed and incubated with blocking buffer followed by mouse anti-MUC5AC $\mathrm{mAb}$ and then NeutrAvidin-HRP (Thermo Pierce Biotechnology) for absorbance at $450 \mathrm{~nm}$. Cell number was determined with $0.5 \%$ crystal violet staining and absorbance at $595 \mathrm{~nm}$. Values for MUC5AC levels were expressed as ratio of absorbance at $450 \mathrm{~nm}$ to $595 \mathrm{~nm}$. For standard-based MUC5AC ELISA, the plasmid encoding the 45M1 epitope of MUC5AC was obtained from G.C. Hansson (Goteburg University, Gothenburg, Sweden), and the epitope was expressed in 293F cells and purified as described previously (65). Protein cell and tissue lysates were plated on Nunc Maxisorp ELISA Plates (Thermo Nalge Nunc) in carbonate-bicarbonate buffer and incubated at $37^{\circ} \mathrm{C}$ overnight. The plates were then washed with PBS-0.05\% Tween-20 (Sigma-Aldrich) and blocked overnight with Sea Block blocking buffer (Thermo Pierce Biotechnology). The reaction was developed using the $45 \mathrm{M} 1$ biotin-conjugated $\mathrm{mAb}$ antibody and avidin-HRP using the 1-Step UltraTMB-Elisa substrate (Thermo Pierce Biotechnology) and assessed for absorbance relative to standard 45M1 concentration.

CLCA1 ELISA. For determinations of CLCA1 in cell lysates, the CLCA1 ELISA was based on rabbit anti-CLCA1 polyclonal antibody raised against $\mathrm{N}$-terminal amino acids 681-693 for detection and purified recombinant CLCA1-3xFLAG containing these residues as standard. For determinations of CLCA1 in cell lysate versus supernatant and in lung tissue samples, the CLCA1 ELISA was based on anti-CLCA1 mAb (clone 8D3) raised against N-terminal CLCA1 amino acids 22-694 and purified N-terminal CLCA1 as standard.

CLCA1-expressing cell lines. To generate lung cell lines that stably expressed CLCA1 driven by the tetracycline-inducible gene promoter system, human lung mucoepidermoid carcinoma NCI-H292 cells were transfected with pCMV-rtTA (rTetR-VP16) (Clonetech), and clones were selected with G418 $(200 \mu \mathrm{g} / \mathrm{ml})$. Clones with low background and high induction of luciferase gene expression when transfected with pTRE-Tight-luciferase (Clonetech) and treated with doxycycline $(10 \mu \mathrm{g} / \mathrm{ml})$ were selected for a second transfection with a TRE-driven CLCA1-expressing plasmid. Cell lines (NCI-H292rtTA-CLCA1 lines) with stable expression of PCMV-rtTA and pTRE-CMVCLCA1 were selected with G418 and hygromycin $(20 \mu \mathrm{g} / \mathrm{ml})$. The CLCA1 gene was cloned from hTEC RNA and was inserted into pTRE-Tight using $5^{\prime}-\mathrm{NotI}$ and $3^{\prime}-\mathrm{EcorV}$ restriction enzyme sites. All constructs were verified by DNA sequencing.

MAPK phospho-arrays. MAPK activation was assessed using a human phospho-MAPK antibody array (Proteome Profiler MAPK Array, R\&D Systems) according to the manufacturer's instructions. The chemiluminescent signal from the arrays was captured on film, digitized with a PowerLook 1120 scanner (UMAX) using SilverFast Ai software (LaserSoft Imaging), and quantified with ArrayVision 8.0 software (GE Healthcare Life Sciences).

Gene knockdown with siRNA. MAPK13 or MAPK14 Stealth RNAi (Life Technologies) was transfected into NCI-H292-rtTA-CLCA1 cells using Lipofectamine RNAiMax (Life Technologies). Cells were then incubated in the RNAi-Lipofectamine mixture for 24 hours at $37^{\circ} \mathrm{C}$ and then with doxycycline for an additional 24 hours. Cellular RNA was isolated and reversed transcribed using the High-Capacity cDNA Archive Kit (Life Technologies), and target mRNA levels were determined by qPCR assay.

Human lung samples. Human tissue was obtained from lung explants harvested at the time of lung transplantation from recipients $(n=20)$ with very severe COPD (GOLD stage IV) during the period from 2005-2009 at BarnesJewish Hospital. Control samples were obtained from donors without COPD $(n=11)$ using both lung explants that were otherwise not useable for transplantation and excess lung tissue that was resected for downsizing. For airway-specific analyses of gene and protein expression, computed tomography imaging and gross histologic examination was used to select airway-containing tissue cores in COPD explants $(n=11)$ and frozen lung tissues from donors without COPD $(n=7)$ as described previously (31). Each analysis consisted of samples from recipients with COPD with the same clinical characteristics (as summarized in Supplemental Table 1). All human studies were conducted with protocols approved by the Washington University School of Medicine Institutional Review Board. 
MAPK inbibitor assay. The MAPK13 blocking activity of test compounds was determined using an IMAP assay containing activated MAPK13, FITC-labeled substrate, and test compound. IMAP assays were performed using the linear phase of the rate kinetics. Assay reactions contained 0-100 $\mu \mathrm{M}$ test compound, 5-35 $\mathrm{nM}\left(\mathrm{EC}_{80}\right)$ activated MAPK13, $3 \mu \mathrm{M}\left(\mathrm{K}_{\mathrm{m}, \mathrm{app}}\right)$ ATP, and $100 \mathrm{nM}$ FITC-labeled EGFR peptide substrate (FITC-KRELVERLTPSGEAPNQALLR-NH $\mathrm{N}_{2}$ ). The $\mathrm{IC}_{50}$ values for each compound were determined from the compound concentration versus fluorescence polarization $(\mathrm{mP})$ plot using nonlinear curve fitting.

MAPK crystallization and structure determination. Human MAPK13 (amino acids 1-352) was expressed and purified for crystallization as described in the Supplemental Methods. Crystals were obtained by mixing protein solution (at $10 \mathrm{mg} / \mathrm{ml}$ ) with reservoir solution $(50 \mathrm{mM}$ ammonium tartrate, $18 \%$ PEG 3350) in a 4:1 (protein/reservoir) ratio. These crystals were used to obtain complex structures with compounds by soaking. Briefly, compounds dissolved in DMSO at a concentration of $100 \mathrm{mM}$ were added to crystallization drops at one-tenth volume for a final concentration of $10 \mathrm{mM}$ compound in the drop and allowed to soak for 2 to 4 hours. X-ray diffraction data were collected at Advanced Photon Source beamline 19ID (nonphosphorylated) and Advanced Light Source (ALS) beamline 4.2.2 (compounds 61 and 124). Data were processed using HKL-2000 software (66). The phase problem was solved by molecular replacement using Phaser crystallographic software (67). For nonphosphorylated MAPK13, the probe structure was a deposited structure of MAPK13 from a structural genomics project (PDB code: 3COI). Although our structure displayed similar crystal packing to the previously deposited one, there were noticeable large differences in the C-terminal region revealed in our high-resolution structure (Supplemental Figure 6). The structures of MAPK13 with compounds were isomorphous to the nonphosphorylated structure, so they were determined by rigid body refinement. Compounds were clearly visible in $F_{o}-F_{c}$ difference maps following rigid body refinement (Supplemental Figure 8). Compounds were fit to electron density maps manually using Coot software (68), and refinement was carried out in PHENIX software (69). Ramachandran analysis was as follows (\% favored/\% percent outliers): nonphosphorylated, 95.6/0.6; compound 61, 96.1/0.3; and compound 124, 95.5/0.9. Final coordinates and experimental structure factors were deposited in the RCSB Protein Data Bank with the following codes: 4EXU, 4EYJ, and 4EYM. Data collection and refinement statistics are provided in Supplemental Table 2. All molecular graphics figures were produced using PyMOL software.

MAPK13 small molecule kinetic assay. Kinetics of MAPK13 binding to small molecules was assessed using BioLayer interferometry with an Octet (ForteBio). Super-streptavidin-coated biosensors from ForteBio were used to capture biotinylated MAPK13 onto the surface of the sensor. After reaching baseline, sensors were moved to association step for 300 seconds and then dissociated for 300 seconds. Curves were corrected by a double-referencing technique, using both biotin-coated pins dipped into the experimental wells and a buffer-only reference. MAPK13-inhibitor complex halflives were calculated from dissociation constants determined from global kinetic analysis.

Statistics. Values for gene expression were analyzed using a 1-way or 2-way ANOVA for a factorial experimental design. PCR data were compared by unpaired Student's $t$ test with Welch's correction for unequal variances when appropriate. Inhibitor effects were analyzed using 2-way ANOVA. $P$ values of less than 0.05 were considered significant.

\section{Acknowledgments}

This work was supported by grants from the NIH (NIAID AADCRC U19-AI070489 and NHLBI P01-HL29594, R01-HL073159, CADET P50-HL107183, and K08-HL083095) and the Martin Schaeffer Fund. We thank the staff in the Flow Cytometry Core in the Siteman Cancer Center and the cores in Pulmonary and Critical Care Medicine for outstanding technical support. These cores include the Epithelial Cell Core supported by the Children's Discovery Institute. Structural results were derived from work performed at Argonne National Laboratory (ANL) Structural Biology Center and the ALS, Berkeley, California, USA. ANL is operated by UChicago Argonne LLC for the US DOE, Office of Biological and Environmental Research (DE-AC02-06CH11357). ALS is supported by the Office of Basic Energy Sciences of the US DOE (DE-AC02-05CH11231). Confocal microscopy was derived in part from work in the Microscopy and Digital Imaging Core of the University Research Center for Auditory and Vestibular Studies supported by NIH NICDD P30DC04665. Antibody generation was supported by the Pathology \& Immunology Hybridoma Center and the Rheumatic Diseases Core Center supported by NIH P30AR048335.

Received for publication May 18, 2012, and accepted in revised form September 13, 2012.

Address correspondence to: Michael J. Holtzman, Washington University School of Medicine, Campus Box 8052, 660 South Euclid Avenue, St. Louis, Missouri 63110, USA. Phone: 314.362.8970; Fax: 314.362.9009; E-mail: holtzmanm@wustl.edu.
1. Kuyper LM, et al. Characterization of airway plugging in fatal asthma. Am J Med. 2003; 115(1):6-11.

2. Hogg JC, et al. The nature of small-airway obstruction in chronic obstructive pulmonary disease. NEngl J Med. 2004;350(26):2645-2653.

3. Brito-Mutunayagam R, Appleton SL, Wilson DH, Ruffin RE, Adams RJ. Global initiative for chronic obstructive lung disease stage 0 is associated with excess FEV1 decline in a representative population sample. Chest. 2010;138(3):605-613.

4. Rose MC, Voynow JA. Respiratory tract mucin genes and mucin glycoproteins in health and disease. Physiol Rev. 2006;86(1):245-278.

5. Wills-Karp $M$, et al. Interleukin-13: central mediator of allergic asthma. Science. 1998; 282(5397):2258-2261.

6. Tyner JW, et al. Blocking airway mucous cell metaplasia by inhibiting EGFR antiapoptosis and IL-13 transdifferentiation signals. J Clin Invest. 2006;116(2):309-321.

7. Grayson $\mathrm{MH}$, et al. Induction of high-affinity $\operatorname{IgE}$ receptor on lung dendritic cells during viral infection leads to mucous cell metaplasia. J Exp Med. 2007;204(11):2759-2769.

8. Kim EY, et al. Persistent activation of an innate immune response translates respiratory viral infection into chronic inflammatory lung disease. Nat Med. 2008;14(6):633-640.

9. Kuperman DA, et al. Direct effects of interleukin-13 on epithelial cells cause airway hyperreactivity and mucus overproduction in asthma. Nat Med. 2002;8(8):885-889.

10. Li D, Gallup M, Fan N, Szymkowski DE, Basbaum $\mathrm{CB}$. Cloning of the amino-terminal and 5'-flanking region of the human MUC5AC mucin gene and transcriptional up-regulation by bacterial exoproducts. J Biol Chem. 1998;273(12):6812-6820.

11. Hewson CA, Edbrooke MR, Johnston SL. PMA induces the MUC5AC respiratory mucin in human bronchial epithelial cells, via PKC, EGF/TGF-a, Ras/Raf, MEK, ERK and Sp-1-dependent mechanisms. J Mol Biol. 2004;344(3):683-695.

12. Atherton HC, Jones G, Danahay H. IL-13-induced changes in the goblet cell density of human bronchial epithelial cell cultures: MAP kinase and phosphatidylinositol 3-kinase regulation. Am J Physiol Lung Cell Mol Physiol. 2003;285(3):L730-L739.

13. Kono Y, et al. Sphingosine kinase 1 regulates mucin production via ERK phosphorylation. Pulm Pharmacol Ther. 2010;23(1):36-42.

14. Patel AC, et al. Genetic segregation of airway disease traits despite redundancy of chloride channel calcium-activated (CLCA) family members. Physiol Genomics. 2006;25(3):502-513.

15. Patel AC, et al. Homologous mouse Clca3,5,6 and human $h C L C A 1,2,4$ gene clusters control mucous cell metaplasia. Am J Respir Crit Care Med. 2007;175:A499.

16. Patel AC, Brett TJ, Holtzman MJ. The role of CLCA proteins in inflammatory airway disease. Annu Rev Physiol. 2009;71:425-449.

17. Gibson A, Lewis AP, Affleck K, Aitken AJ, Meldrum E, Thompson N. hCLCA1 and mCLCA3 are secreted non-integral membrane proteins and therefore are not ion channels. J Biol Chem. 
2005;280(29):27205-27212

18. Mundhenk L, Alfalah M, Elble RC, Pauli BU, Naim HY, Gruber AD. Both cleavage products of the mCLCA3 protein are secreted soluble proteins. J Biol Chem. 2006;281(40):30072-30080.

19. Goldstein DM, Kuglstatter A, Lou Y, Soth MJ Selective p38 $\alpha$ inhibitors clinically evaluated for the treatment of chronic inflammatory disorders. J Med Chem. 2010;53(6):2345-2353.

20. Nakano T, et al. Niflumic acid suppresses interleukin-13-induced asthma phenotypes. Am J Respir Crit Care Med. 2006;173(11):1216-1221.

21 . Kettle R, et al. Regulation of neuregulin 1 $1 \beta 1$-induced MUC5AC and MUC5B expression in human airway epithelium. Am J Respir Cell Mol Biol. 2010;42(4):472-481.

22. Kirkham S, Kolsum U, Rousseau K, Singh D, Vestbo J, Thornton DJ. MUC5B is the major mucin in the gel phase of sputum in chronic obstructive pulmonary disease. Am J Respir Crit Care Med. 2008;178(10):1033-1039.

23. Sepper R, et al. Mucin5B expression by lung alveolar macrophages is increased in long-term smokers. J Leukoc Biol. 2012;92(2):319-324.

24. Leverkoehne I, Gruber AD. The murine mCLCA3 (alias gob-5) protein is located in the mucin granule membranes of intestinal, respiratory, and uterine goblet cells. J Histochem Cytochem. 2002;50(6):829-838.

25. Raiford KL, Park J, Lin K, Fang S, Crews AL, Adler $\mathrm{KB}$. Mucin granule-associated proteins in human bronchial epithelial cells: the airway goblet cell "granulome". Respir Res. 2011;12:118.

26 . Song KS, et al. Interleukin-1b and tumor necrosis factor-a induce MUC5AC overexpression through a mechanism involving ERK/p38 mitogen-activated protein kinases-MSK1-CREB activation in human airway epithelial cells. J Biol Chem. 2003;278(26):23243-23250.

27. Karaman MW, et al. A quantitative analysis of kinase inhibitor selectivity. Nat Biotechnol. 2008;26(1):127-132.

28. Yuan-chen Wu D, Wu R, Reddy SP, Lee YC, Chang MM. Distinctive epidermal growth factor receptor/ extracellular regulated kinase-independent and -dependent signaling pathways in the induction of airway mucin 5B and mucin 5AC expression by phorbol 12-myristate 13-acetate. Am J Pathol. 2007;170(1):20-32.

29. Kuma Y, Sabio G, Bain J, Shprio N, Marquez $\mathrm{R}$, Cuenda A. BIRB796 inhibits all p38 MAPK isoforms in vitro and in vivo. I Biol Chem 2005;280(20):19472-19479.

30. Cuenda A, et al. SB203580 is a specific inhibitor of a MAP kinase homologue which is stimulated by cellular stresses and interleukin-1. FEBS Lett. 1995;364(2):229-233.

31. Deslee $G$, et al. Cigarette smoke induces nucleic acid oxidation in lung fibroblasts. Am J Respir Cell Mol Biol. 2010;43(5):576-584.

32. Sabio $G$, et al. p38g regulates the localisation of SAP97 in the cytoskeleton by modulating its interaction with GKAP. EMBOJ. 2005;24(6):1134-1145.

33. Boehm H-J, Flohr A, Stahl M. Scaffold hopping. Drug Discov Today. 2004;1(3):217-224.

34. Sun H, Tawa G, Wallqvist A. Classification of scaffold-hopping approaches. Drug Discov Today. 2012;17(7-8):310-324
35. Pargellis C, et al. Inhibition of p38 MAP kinase by utilizing a novel allosteric binding site. Nat Struct Biol. 2002;9(4):268-272.

36. Kuglstatter A, et al. X-ray crystal structure of JNK2 complexed with the $\mathrm{p} 38 \alpha$ inhibitor BIRB796: insights into the rational design of DFG-out binding MAP kinase inhibitors. Bioorg Med Chem Lett. 2010;20(17):5217-5220.

37. Liu Y, Gray NS. Rational design of inhibitors that bind to inactive kinase conformations. Nat Chem Biol. 2006;2(7):358-364

38. Lee MR, Dominguez C. MAP kinase inhibitors: clinical results and an intimate look at their interactions with $\mathrm{p} 38 \alpha$ protein. Curr Med Chem. 2005;12(25):2979-2994.

39. Huan C, et al. mCLCA4 ER processing and secretion requires luminal sorting motifs. Am J Physiol. 2008;295(1):C279-C287.

40. Hamann M, et al. Human Clca1 modulates anionic conduction of calcium-dependent chloride currents. J Physiol. 2009;587(pt 10):2255-2274.

41. Bothe MK, Braun J, Mundhenk L, Gruber AD. Murine mCLCA6 is an integral apical membrane protein of non-goblet cell enterocytes and co-localizes with the cystic fibrosis transmembrane conductance regulator. $J$ Histochem Cytochem. 2008;56(5):495-509.

42. Braun J, Bothe MK, Mundhenk L, Beck CL, Gruber AD. Murine mCLCA5 is expressed in granular layer keratinocytes of stratified epithelia. Histochem Cell Biol. 2010;133(3):285-299.

43. Bothe MK, Mundhenk L, Kaup M, Weise C, Gruber $\mathrm{AD}$. The murine goblet cell protein mCLCA3 is a zinc-dependent metalloprotease with autoproteolytic activity. Mol Cells. 2011;32(6):535-541.

44. Bothe MK, Mundhenk L, Beck CL, Kaup M, Gruber AD. Impaired autoproteolytic cleavage of mCLCA6, a murine integral membrane protein expressed in enterocytes, leads to cleavage at the plasma membrane instead of the endoplasmic reticulum. Mol Cells. 2012;33(3):251-257.

45. Yurtsever $Z$, et al. Self-cleavage of human CLCA1 by a novel internal metalloprotease domain controls calcium-activated chloride channel activation [published online ahead of print October 30, 2012].J Biol Chem . doi: 10.1074/jbc.M112.410282

46. Cargnello M, Roux PP. Activation and function of the MAPKs and their substrates, the MAP$\mathrm{K}$-activated protein kinases. Microbiol Mol Biol Rev. 2011;75(1):50-83

47. Cuadrado A, Nebreda AR. Mechanisms and functions of p38 MAPK signalling. Biochem J. 2010;429(3):403-417.

48. Duvvuri U, et al. TMEM16A induces MAPK and contributes directly to tumorigenesis and cancer progression. Cancer Res. 2012;72(13):3270-3281.

49. Jiang $Y$, et al. Characterization of the structure and function of the fourth member of p38 group mitogen-activated protein kinases, p38delta. J Biol Chem. 1997;272(48):30122-20128.

50. Eckert RL, Efimova T, Balasubramanian S, Crish JF, Bone F, Dashti S. p38 mitogen-activated protein kinases on the body surface-a function for $\mathrm{p} 38 \mathrm{~d}$. J Invest Dermatol. 2003;120(5):823-828.

51. Effimova T, Broome AM, Eckert RL. Protein kinase Cdelta regulates keratinocyte death and survivial by regulating activity and subcellular localization of a p38delta-extracellular signal-regulated kinase
1/2 complex. Mol Cell Biol. 2004;24(18):8167-8183.

52. Feijoo C, Campbell DG, Jakes R, Goedert M, Cuenda A. Evidence that phosphorylation of the microtubule-associated protein Tau by SAPK4/ p38delta at Thr50 promotes microtubule assembly. J Cell Sci. 2005;118(pt 2):397-408

53. Sumara G, et al. Regulation of PKD by the MAPK p38 in insulin secretion and glucose homeostasis. Cell. 2009;136(2):235-248.

54. Tan FL, et al. p38delta/MAPK13 as a diagnostic marker for cholangiocarcinoma and its involvement in cell motility and invasion. Int J Cancer. 2009;126(10):2353-2361.

55. Schindler E, et al. p38delta Mitogen-activated protein kinase is essential for skin tumor development in mice. Cancer Res. 2009;69(11):4648-4655.

56. Renda $\mathrm{T}$, et al. Increased activation of $\mathrm{p} 38 \mathrm{MAPK}$ in COPD. Eur Respir J. 2008;31(1):62-69.

57. Medicherla S, et al. p38a-selective mitogen-activated protein kinase inhibitor SD-282 reduces inflammation in a subchronic model of tobacco smoke-induced airway inflammation. J Pharmacol Exp Ther. 2008;324(3):921-929.

58. Risco A, et al. p38g and p38d kinases regulate the Toll-like receptor 4 (TLR4)-induced cytokine production by controlling ERK1/2 protein kinase pathway activation. Proc Natl Acad Sci U S A. 2012;109(28):11200-11205

59. Soth M, et al. 3-Amino-pyrazolo[3,4-d]pyrimidines as p38 $\alpha$ kinase inhibitors: design and development to a highly selective lead. Bioorg Med Chem Lett. 2011;21(11):3452-3456

60. Koeberle SC, et al. Skepinone-L is a selective p38 mitogen-activated protein kinase inhibitor. Nat Chem Biol. 2011;8(2):141-143.

61. Chung KF. p38 mitogen-activated protein kinase pathways in asthma and COPD. Chest. 2011;139(6):1470-1479

62. Bannerjee A, Koziol-White C, Panettieri RJ p38 MAPK inhibitors, IKK2 inhibitors, and TNFa inhibitors in COPD. Curr Opin Pharmacol. 2012;12(3):287-292.

63. Thavagnanam S, Parker JC, McBrien ME, Skibinski G, Heaney LG, Shields MD. Effects of IL-13 on mucociliary differentiation of pediatric asthmatic bronchial epithelial cells. Ped Res. 2011;69(2):95-100.

64. Stewart SA, et al. Stable silencing of gene expression in primary cells by retroviral delivery of RNAi. RNA. 2003;9(4):493-501.

65. Lidell ME, Bara J, Hansson GC. Mapping of the $45 \mathrm{M} 1$ epitope to the C-terminal cysteine-rich part of the human MUC5AC mucin. FEBS J. 2008;275(3):481-489.

66. Otwinowski Z, Minor W. Processing of x-ray diffraction data collected in oscillation mode. In: Carter CWJ, Sweet RM, eds. Methods in Enzymology: Macromolecular Crystallography. New York, New York, USA: Academic Press; 1997:307-326.

67. McCoy AJ, Grosse-Kunstleve RW, Adams PD, Winn $\mathrm{MD}$, Storoni LC, Read RJ. Phaser crystallographic software. J Appl Crystallogr. 2007;40(pt 4):658-674.

68. Emsley P, Lohkamp B, Scott WG, Cowtan K. Features and development of Coot. Acta Crystallogr D Biol Crystallogr. 2010;66(pt 4):486-501.

69. Adams PD, et al. PHENIX: a comprehensive Python-based system for macromolecular structure solution. Acta Crystallogr D Biol Crystallogr. 2010;66(pt 2):213-221. 